\title{
Dark sector assisted low scale leptogenesis from three body decay
}

\author{
Debasish Borah $\odot,{ }^{1, *}$ Arnab Dasgupta, ${ }^{2, \dagger}$ and Devabrat Mahanta ${ }^{1, \ddagger}$ \\ ${ }^{1}$ Department of Physics, Indian Institute of Technology Guwahati, Assam 781039, India \\ ${ }^{2}$ Institute of Convergence Fundamental Studies, Seoul-Tech, Seoul 139-743, Korea
}

(Received 9 November 2020; revised 1 November 2021; accepted 24 December 2021; published 12 January 2022)

\begin{abstract}
We study the possibility of realizing leptogenesis from three body decay, dark matter (DM) and neutrino mass in a minimal framework. We propose a first of its kind model to implement the idea of leptogenesis from three body decay where $C P$ asymmetry arises from interference of multiple $1 \rightarrow 3$ diagrams using resummed propagators along with DM. The standard model is extended by three heavy singlet fermions, one scalar singlet, and one scalar doublet with appropriate discrete charges. Two of these singlet fermions not only play nontrivial roles in generating light neutrino mass at radiative level in scotogenic fashion, but also act as mediators in three body decay of the third singlet fermion leading to desired $C P$ asymmetry through interference of such diagrams. With just one additional field compared to the minimal scotogenic model, we show that successful leptogenesis can occur at a scale as low as approximately $1 \mathrm{TeV}$ which is much lower than the leptogenesis scale found for minimal scotogenic model. Also, the realization of this three body decay leptogenesis naturally leads to a two component scalar singlet-doublet dark matter scenario offering a rich phenomenology. Apart from having interesting interplay of different couplings involved in processes related to both leptogenesis and dark matter, the model can also be tested at different experiments due to the existence of its particle spectrum at $\mathrm{TeV}$ scale.
\end{abstract}

DOI: 10.1103/PhysRevD.105.015015

\section{INTRODUCTION}

The observed asymmetry between matter and antimatter in the present universe has been a longstanding puzzle in particle physics and cosmology. The excess of baryon over antibaryons is so huge that almost all the visible matter in the universe is in the form of baryons only. It is often quantified in terms of baryon to photon ratio, which, according to Planck 2018 data $[1,2]$ is

$$
\eta_{B}=\frac{n_{B}-n_{\bar{B}}}{n_{\gamma}}=6.1 \times 10^{-10} .
$$

This excess derived from the measurements of cosmic microwave background (CMB) anisotropies matches very well with the predictions of big bang nucleosynthesis (BBN). The observed excess gives rise to a puzzle because we expect the universe to be started in a baryon symmetric manner. Even if we start with an initial asymmetry, the cosmic inflationary phase will make it negligible in latter

\footnotetext{
*dborah@iitg.ac.in

†arnabdasgupta@protonmail.ch

*devab176121007@iitg.ac.in
}

Published by the American Physical Society under the terms of the Creative Commons Attribution 4.0 International license. Further distribution of this work must maintain attribution to the author(s) and the published article's title, journal citation, and DOI. Funded by SCOAP. epochs of the universe. A baryon symmetric universe can evolve into an asymmetric one dynamically if certain conditions, known as Sakharov's conditions [3] are satisfied. They are namely, (1) baryon number (B) violation, (2) $C$ and $C P$ violation and (3) departure from thermal equilibrium. While all these criteria can be satisfied, in principle, in the standard model (SM) of particle physics and an expanding Friedman-Lemaitre-Robertson-Walker (FLRW) universe, it falls way short of the required amount to produce the huge asymmetry. This has led to different beyond standard model (BSM) proposals out of which the most popular scenario is to consider the existence of some heavy particles whose out-of-equilibrium and $\mathrm{B}, C, C P$ violating decays can produce the baryon asymmetry of the universe (BAU) [4,5]. Instead of generating baryon asymmetry directly, one can generate an asymmetry in the leptonic sector first through similar lepton number (L) violating decays which can later be converted into the observed baryon asymmetry through $(B+L)$-violating EW sphaleron transitions [6]. First proposed by Fukugita and Yanagida more than thirty years back [7], this alternate way has come to be known as leptogenesis, a review of which can be found in [8]. An interesting feature of this scenario is that the required lepton asymmetry can be generated through $C P$ violating out-of-equilibrium decays of the same heavy fields that take part in the seesaw mechanism [9-14] that explains the origin of tiny neutrino masses [1], another observed phenomenon the SM fails to address. 
While the asymmetric nature of visible matter has been a longstanding puzzle, another feature of the overall matter component of the present universe adds more to this puzzle. It turns out that only approximately $20 \%$ of the present universe's matter density is composed of baryons or visible matter while the rest comes from a mysterious, nonluminous, nonbaryonic form of matter, popularly known as dark matter (DM). This is strongly supported by both astrophysical and cosmological observations [1,2,15-17]. Similar to the baryon asymmetry, DM abundance is also quantified in terms of a dimensionless quantity as [2]: $\Omega_{\mathrm{DM}} h^{2}=0.120 \pm 0.001$ at $68 \%$ confidence level (CL). Here $\Omega_{\mathrm{DM}}=\rho_{\mathrm{DM}} / \rho_{\text {critical }}$ is the density parameter of $\mathrm{DM}$ and $h=$ Hubble Parameter $/\left(100 \mathrm{~km} \mathrm{~s}^{-1} \mathrm{Mpc}^{-1}\right)$ is a dimensionless parameter of order unity. $\rho_{\text {critical }}=$ $3 H^{2} /(8 \pi G)$ is the critical density while $H$ is the Hubble parameter. Since none of the SM particles can satisfy the criteria for being a DM candidate, several BSM proposals have been put forward out of which the weakly interacting massive particle (WIMP) is the most widely studied one $[18,19]$. In the WIMP framework, a DM particle having mass around the electroweak scale and interactions similar to the weak interactions gets thermally produced in the early universe, followed by its decoupling from the thermal bath leading to a freeze-out abundance remarkably close to the observed DM abundance. This coincidence is often referred to as the WIMP miracle.

Motivated by the above two phenomena and neutrino mass which SM fails to explain, we consider a scenario where all three phenomena can be explained in a unified manner. One popular scenario, which can accommodate all these three phenomena is the scotogenic framework proposed by $\mathrm{Ma}$ in 2006 [20]. In the minimal version of this framework, the SM is extended by two or three copies of $Z_{2}$ odd fermions singlet under SM gauge symmetries, and an additional scalar field similar to the Higgs doublet of the $\mathrm{SM}$, but odd under the unbroken $Z_{2}$ symmetry. The salient feature of this framework is the way it connects the origin of light neutrino masses and DM. The unbroken $Z_{2}$ symmetry leads to a stable DM candidate while the $Z_{2}$ odd particles generate light neutrino masses at one loop level. Apart from this, the out-of-equilibrium decay of the heavy singlet fermions can also lead to successful leptogenesis at a scale as low as $10 \mathrm{TeV}$. Such low scale leptogenesis with hierarchical right handed neutrinos has been discussed by several authors [21-28] while quasidegenerate right handed neutrino scenario was discussed in earlier works [29,30]. For hierarchical right handed neutrinos, such a low scale leptogenesis is a significant improvement over the usual Davidson-Ibarra bound $M_{1}>10^{9} \mathrm{GeV}$ for vanilla leptogenesis in type I seesaw framework [31].

In this work, we consider the possibility of lowering the scale of leptogenesis further (compared to the ones obtained in previous works) via decay heavy singlet fermions into three different particles including one SM lepton. While leptogenesis from three body decay was covered in earlier works [32-35], a different way of achieving the same was also discussed in different contexts by the authors of [36-40]. These works either considered soft leptogenesis in a supersymmetric framework or leptogenesis due to $C P$ asymmetry arising from interference of from multiple $2 \rightarrow 2$ or $1 \rightarrow n(n \geq 3)$ diagrams with resummed propagators. While a concrete model to realize leptogenesis from such $2 \rightarrow 2$ processes along with dark matter was proposed in [36], there has been no concrete model yet, as far as we are aware of, to realize leptogenesis from three body decay where nonzero $C P$ asymmetry arises due to interference of multiple $1 \rightarrow 3$ decay diagrams with resummed propagators together with dark matter. Here we try to implement this idea in a minimal extension of the scotogenic model to achieve successful leptogenesis at a low scale. Such an extension is required as in the minimal scotogenic model we can not have nonzero $C P$ asymmetry from three body decay of right handed neutrinos. We show that successful leptogenesis can be achieved at a scale as low as $2 \mathrm{TeV}$ in this scenario, which gets lowered to even below $1 \mathrm{TeV}$ after including the lepton flavor effects. While, the usual two body decay of right handed neutrinos in the scotogenic model can also contribute to lepton asymmetry, we show that the contribution from three body decay dominates in the low mass regime. While building such a setup, we also find that the model naturally predicts a two component dark matter scenario. We discuss interplay of different couplings involved in leptogenesis as well dark matter and show the consistency between the possibility of low scale leptogenesis and correct DM relic density in agreement with all experimental constraints including light neutrino masses and mixing.

This paper is organized as follows. In Sec. II, we discuss our model in details followed by discussion of leptogenesis and dark matter in Secs. III and IV respectively. We discuss our results in Sec. V and conclude in Sec. VI.

\section{THE MODEL}

We briefly discuss our model in this section. We stick to a minimal setup required to obtain the desired phenomenology. The SM particle content is extended by three singlet chiral fermions $N_{1,2}, \psi$ and two scalar fields $\eta, S$ which transform nontrivially under the additional $Z_{2} \times Z_{2}^{\prime}$ symmetry of the model. This additional discrete symmetry is chosen to remove the unwanted terms so that the desired leptogenesis and dark matter phenomenology can be ensured. UV completion of our model can explain the origin of such discrete gauge symmetries from spontaneous breaking of gauge symmetries at high energy scale, for example see [41-46] and references therein. As we discuss in details below, with the addition of one extra field compared to the minimal scotogenic model [20], we can achieve much richer phenomenology with a new way of 
TABLE I. Particle content of the model.

\begin{tabular}{lccc}
\hline \hline Particles & $S U(3)_{c} \times S U(2)_{L} \times U(1)_{Y}$ & $Z_{2}$ & $Z_{2}^{\prime}$ \\
\hline$Q_{L}$ & $\left(3,2, \frac{1}{6}\right)$ & 1 & 1 \\
$u_{R}$ & $\left(3,1, \frac{2}{3}\right)$ & 1 & 1 \\
$d_{R}$ & $\left(3,1,-\frac{1}{3}\right)$ & 1 & 1 \\
$\ell_{L}$ & $\left(1,2,-\frac{1}{2}\right)$ & 1 & 1 \\
$\ell_{R}$ & $(1,1,-1)$ & 1 & 1 \\
$N_{1,2}$ & $(1,1,0)$ & -1 & 1 \\
$\psi$ & $(1,1,0)$ & -1 & -1 \\
$H$ & $\left(1,2, \frac{1}{2}\right)$ & 1 & 1 \\
$\eta$ & $\left(1,2, \frac{1}{2}\right)$ & -1 & 1 \\
$S$ & $(1,1,0)$ & 1 & -1 \\
\hline \hline
\end{tabular}

generating lepton asymmetry at a scale, lower compared to the one obtained in minimal scotogenic model [24-28].

The particle content of the model is shown in Table I along with their transformations under the symmetries of the model. The Yukawa Lagrangian can be written as

$$
\begin{aligned}
\mathcal{L}= & -Y_{u} \overline{Q_{L}} \tilde{H} u_{R}-Y_{d} \overline{Q_{L}} H d_{R}-Y_{e} \overline{\ell_{L}} H \ell_{R}-h_{i \alpha}\left(\bar{\ell}_{L}\right)_{i} \tilde{\eta} N_{\alpha} \\
& -\frac{1}{2} M_{\alpha} \overline{N_{\alpha}^{c}} N_{\alpha}-y_{\alpha} \psi N_{\alpha} S-\frac{1}{2} m_{\psi} \overline{\psi^{c}} \psi
\end{aligned}
$$

The scalar potential is given by

$$
\begin{aligned}
V= & \mu_{H}^{2} H^{\dagger} H+\mu_{\eta}^{2} \eta^{\dagger} \eta+\frac{1}{2} m^{2} S^{2}+\frac{\lambda_{1}}{2}\left(H^{\dagger} H\right)^{2}+\frac{\lambda_{2}}{2}\left(\eta^{\dagger} \eta\right)^{2} \\
& +\frac{1}{2} \lambda_{S} S^{4}+\lambda_{3}\left(H^{\dagger} H\right)\left(\eta^{\dagger} \eta\right)+\lambda_{4}\left(H^{\dagger} \eta\right)\left(\eta^{\dagger} H\right) \\
& +\frac{\lambda_{5}}{2}\left[\left(H^{\dagger} \eta\right)\left(H^{\dagger} \eta\right)+\left(\eta^{\dagger} H\right)\left(\eta^{\dagger} H\right)\right] \\
& +\frac{\lambda_{6}}{2}\left(H^{\dagger} H\right) S^{2}+\lambda_{7}\left(\eta^{\dagger} \eta\right) S^{2} .
\end{aligned}
$$

After the electroweak symmetry breaking (EWSB), the two scalar doublets of the model can be written in the following form in the unitary gauge:

$$
H=\left(\begin{array}{c}
0 \\
\frac{v+h}{\sqrt{2}}
\end{array}\right), \quad \eta=\left(\begin{array}{c}
\eta^{ \pm} \\
\frac{\eta_{R}+i \eta_{I}}{\sqrt{2}}
\end{array}\right),
$$

where $h$ is the SM-like Higgs boson, $\eta_{R}, \eta_{I}$ are the $C P$-even and $C P$-odd neutral scalars respectively, while $\eta^{ \pm}$are the charged scalars from the additional scalar doublet $\eta$. The vacuum expectation value (VEV) of the SM Higgs is denoted by $v$ while the other two scalars do not acquire any VEVs so that the $Z_{2} \times Z_{2}^{\prime}$ symmetry of the model remains unbroken.

The masses of the physical scalars at tree level can be written as

$$
\begin{aligned}
& m_{h}^{2}=\lambda_{1} v^{2}, \quad m_{S}^{2}=m^{2}+\lambda_{6} \frac{v^{2}}{2}, \quad m_{\eta^{ \pm}}^{2}=\mu_{\eta}^{2}+\frac{1}{2} \lambda_{3} v^{2} \\
& m_{\eta_{R}}^{2}=\mu_{\eta}^{2}+\frac{1}{2}\left(\lambda_{3}+\lambda_{4}+\lambda_{5}\right) v^{2}=m_{\eta^{ \pm}}^{2}+\frac{1}{2}\left(\lambda_{4}+\lambda_{5}\right) v^{2}, \\
& m_{\eta_{I}}^{2}=\mu_{\eta}^{2}+\frac{1}{2}\left(\lambda_{3}+\lambda_{4}-\lambda_{5}\right) v^{2}=m_{\eta^{ \pm}}^{2}+\frac{1}{2}\left(\lambda_{4}-\lambda_{5}\right) v^{2} .
\end{aligned}
$$

Without any loss of generality, we consider $\lambda_{5}<0$ so that the $C P$-even scalar is lighter than the $C P$-odd one. Thus $\eta_{R}$ is the lightest component of the scalar doublet $\eta$ and also lighter than the singlet fermions $N_{1,2}$. Similarly the singlet scalar $S$ is chosen to be lighter than $\psi$. This ensures $\eta_{R}, S$ to be the lightest $Z_{2}$-odd and $Z_{2}^{\prime}$-odd particles respectively and hence viable dark matter candidates of the model.

As can be seen from the Yukawa Lagrangian in Eq. (2), there is no tree level contribution to light neutrino masses, simply because they couple to the heavy neutrinos $N_{i}$ only via the second scalar doublet $\eta$ which does not acquire any VEV. However, light neutrino masses can arise at radiative level as originally proposed in the context of minimal scotogenic model [20]. In our setup, the additional scalar doublet $\eta$ and the singlet fermions $N_{1,2}$ will go inside the loop which generates the light neutrino masses, the expression for which can be evaluated as $[20,47]$

$$
\begin{aligned}
\left(M_{\nu}\right)_{i j} & =\sum_{k} \frac{h_{i k} h_{j k} M_{k}}{32 \pi^{2}}\left(\frac{m_{\eta_{R}}^{2}}{m_{\eta_{R}}^{2}-M_{\alpha}^{2}} \ln \frac{m_{\eta_{R}}^{2}}{M_{k}^{2}}-\frac{m_{\eta_{I}}^{2}}{m_{\eta_{I}}^{2}-M_{k}^{2}} \ln \frac{m_{\eta_{I}}^{2}}{M_{k}^{2}}\right) \\
& \equiv \sum_{k} \frac{h_{i k} h_{j k} M_{k}}{32 \pi^{2}}\left[L_{k}\left(m_{\eta_{R}}^{2}\right)-L_{k}\left(m_{\eta_{I}}^{2}\right)\right]
\end{aligned}
$$

where $M_{k}$ is the mass eigenvalue of the mass eigenstate $N_{k}$ in the internal line and the indices $i, j=1,2,3$ run over the three neutrino generations and $k=1,2$ takes into account of two $N_{\alpha}$. The loop function $L_{k}\left(m^{2}\right)$ is defined as

$$
L_{k}\left(m^{2}\right)=\frac{m^{2}}{m^{2}-M_{k}^{2}} \ln \frac{m^{2}}{M_{k}^{2}} .
$$

From the expressions for physical scalar masses given in Eqs. (5), we can write $m_{\eta_{R}}^{2}-m_{\eta_{I}}^{2}=\lambda_{5} v^{2}$. Therefore, in the limit $\lambda_{5} \rightarrow 0$, the neutral components of inert doublet $\eta$ become mass degenerate. Also, a vanishing $\lambda_{5}$ implies vanishing light neutrino masses which is expected as the $\lambda_{5^{-}}$ term in the scalar potential (3) breaks lepton number by two units, when considered together with the fermion Yukawa Lagrangian (2). As we will see later, this parameter also plays crucial role in both leptogenesis and DM phenomenology. It should also be noted that the Yukawa coupling $h_{i k}$ is a $3 \times 2$ matrix in flavor basis due to the existence of only two right handed neutrinos appearing in light neutrino mass. This predicts a vanishing lightest neutrino mass.

In order to ensure that the choice of Yukawa couplings as well as other parameters involved in light neutrino mass formula discussed above are consistent with the 
cosmological upper bound on the sum of neutrino masses, $\sum_{i} m_{i} \leq 0.11 \mathrm{eV}$ [2], as well as the neutrino oscillation data $[48,49]$, it is often useful to rewrite the neutrino mass formula given in Eqs. (6) in a form similar to the well known the type-I seesaw formula:

$$
M_{\nu}=h \Lambda^{-1} h^{T},
$$

where we have introduced the diagonal matrix $\Lambda$ with elements

$$
\Lambda_{\alpha}=\frac{2 \pi^{2}}{\lambda_{5}} \zeta_{\alpha} \frac{2 M_{\alpha}}{v^{2}}
$$

and $\zeta_{\alpha}=\left(\frac{M_{\alpha}^{2}}{8\left(m_{\eta_{R}}^{2}-m_{\eta_{I}}^{2}\right)}\left[L_{\alpha}\left(m_{\eta_{R}}^{2}\right)-L_{\alpha}\left(m_{\eta_{I}}^{2}\right)\right]\right)^{-1}$.

The light neutrino mass matrix (8) which is complex symmetric by virtue of Majorana nature, can be diagonalized by the usual Pontecorvo-Maki-Nakagawa-Sakata (PMNS) mixing matrix $U$ (in the diagonal charged lepton basis), written in terms of neutrino oscillation data (up to the Majorana phases) as

$$
U=\left(\begin{array}{ccc}
c_{12} c_{13} & s_{12} c_{13} & s_{13} e^{-i \delta} \\
-s_{12} c_{23}-c_{12} s_{23} s_{13} e^{i \delta} & c_{12} c_{23}-s_{12} s_{23} s_{13} e^{i \delta} & s_{23} c_{13} \\
s_{12} s_{23}-c_{12} c_{23} s_{13} e^{i \delta} & -c_{12} s_{23}-s_{12} c_{23} s_{13} e^{i \delta} & c_{23} c_{13}
\end{array}\right) U_{\text {Maj }}
$$

where $c_{i j}=\cos \theta_{i j}, s_{i j}=\sin \theta_{i j}$ and $\delta$ is the leptonic Dirac $C P$ phase. The diagonal matrix $U_{\text {Maj }}=\operatorname{diag}\left(1, e^{i \alpha}, e^{i(\zeta+\delta)}\right)$ contains the undetermined Majorana $C P$ phases $\alpha$, $\zeta$. The diagonal light neutrino mass matrix is therefore,

$$
D_{\nu}=U^{\dagger} M_{\nu} U^{*}=\operatorname{diag}\left(m_{1}, m_{2}, m_{3}\right) .
$$

where the light neutrino masses can follow either normal ordering (NO) or inverted ordering (IO). As mentioned earlier, the model predicts a vanishing lightest neutrino mass implying $m_{1}=0(\mathrm{NO})$ and $m_{3}=0$ (IO). Since the inputs from neutrino oscillation data are only in terms of the two mass squared differences and three mixing angles, it would be useful for our purpose to express the Yukawa couplings $(h)$ in terms of light neutrino parameters. This is possible through the Casas-Ibarra (CI) parametrization [50] extended to radiative seesaw model [51] which allows us to write the Yukawa coupling matrix satisfying the neutrino data as

$$
h_{i \alpha}=\left(U D_{\nu}^{1 / 2} R^{\dagger} \Lambda^{1 / 2}\right)_{i \alpha},
$$

where $R$ is an arbitrary complex orthogonal matrix satisfying $R R^{T}=\nVdash$. It is worth mentioning that, since we have only two right handed neutrinos $N_{1,2}$ taking part in generating radiative light neutrino masses, the lightest neutrino mass is vanishing. Also, in case of only two right handed neutrinos, the $R$ matrix is a function of only one complex rotation parameter $z=z_{R}+i z_{I}, z_{R} \in[0,2 \pi]$, $z_{I} \in \mathbb{R}$ [52] which can affect the results of leptogenesis as we discuss below.

\section{A. Constraints on model parameters}

Precision measurements at LEP experiment forbids additional decay channels of the SM gauge bosons. For example, it strongly constrains the decay channel $Z \rightarrow \eta_{R} \eta_{I}$ requiring $m_{\eta_{R}}+m_{\eta_{I}}>m_{Z}$. Additionally, LEP precision data also rule out the region $m_{\eta_{R}}<80 \mathrm{GeV}, m_{\eta_{I}}<$ $100 \mathrm{GeV}, m_{\eta_{I}}-m_{\eta_{R}}>8 \mathrm{GeV}$ [53]. We take the lower bound on charged scalar mass $m_{\eta^{ \pm}}>90 \mathrm{GeV}$. If $m_{\eta_{R}, \eta_{I}}<$ $m_{h} / 2$, the large hadron collider (LHC) bound on invisible Higgs decay comes into play. The constraint on the Higgs invisible decay branching fraction from the ATLAS experiment at LHC is [54]

$\mathcal{B}(h \rightarrow$ Invisible $)=\frac{\Gamma(h \rightarrow \text { Invisible })}{\Gamma(h \rightarrow S M)+\Gamma(h \rightarrow \text { Invisible })} \leq 26 \%$

while the recent ATLAS announcement [55] puts a more stringent constraint at $13 \%$. This can constrain the SM Higgs coupling with $\eta_{R}, \eta_{I}, S$ namely $\lambda_{3}+\lambda_{4} \pm \lambda_{5}, \lambda_{6}$ respectively to be smaller than around $10^{-3}$ in the regime $m_{\eta_{R}}, m_{\eta_{I}}, m_{S}<m_{h} / 2$ which however remains weaker than DM direct detection bounds in this mass regime (see for example, [56]).

Additionally, the LHC experiment can also put bounds on the scalar masses in the model, specially the components of scalar doublet $\eta$ as they can be pair produced copiously in proton collisions leading to different final states which are being searched for. Depending upon the mass spectrum of its components, the heavier ones can decay into the lighter ones and a gauge boson, which finally decays into a pair of leptons or quarks. Therefore, we can have either pure leptonic final states plus missing transverse energy 
(MET), hadronic final states plus MET or a mixture of both. The MET corresponds to DM or light neutrinos. In several earlier works [57-59], the possibility of opposite sign dileptons plus MET was discussed. In [60], the possibility of dijet plus MET was investigated with the finding that inert scalar masses up to $400 \mathrm{GeV}$ can be probed at high luminosity LHC. In another work [61], trilepton plus MET final states was also discussed whereas monojet signatures have been studied by the authors of $[62,63]$. The enhancement in dilepton plus MET signal in the presence of additional vectorlike singlet charged leptons was also discussed in [64]. Exotic signatures like displaced vertex and disappearing or long-lived charged track for compressed mass spectrum of inert scalars and singlet fermion DM was studied recently by the authors of [65].

In addition to the collider or direct search constraints, there exists theoretical constraints also. For instance, the scalar potential of the model should be bounded from below in any field direction. This criteria leads to the following copositivity conditions [66-68]:

$$
\begin{array}{cl}
\lambda_{1,2, S} \geq 0, & \lambda_{3}+\sqrt{\lambda_{1} \lambda_{2}} \geq 0, \\
\lambda_{3}+\lambda_{4}-\left|\lambda_{3}\right|+\sqrt{\lambda_{1} \lambda_{2}} \geq 0, & \lambda_{6}+2 \sqrt{\lambda_{1} \lambda_{S}} \geq 0, \\
\sqrt{\lambda_{1} \lambda_{2} \lambda_{S}}+2\left(\lambda_{3} \sqrt{\lambda_{S}}+\lambda_{7} \sqrt{\lambda_{1}}+\frac{\lambda_{6}}{2} \sqrt{\lambda_{2}}\right)+4 \sqrt{\frac{1}{2}\left(\lambda_{3}+\frac{1}{2} \sqrt{\lambda_{1} \lambda_{2}}\right)\left(\lambda_{6}+\sqrt{\lambda_{1} \lambda_{S}}\right)\left(\lambda_{7}+\frac{1}{2} \sqrt{\lambda_{2} \lambda_{6}}\right)} \geq 0 .
\end{array}
$$

The coupling constants appeared in above expressions are evaluated at the electroweak scale, $v$. Also, in order to avoid perturbative breakdown, all dimensionless couplings like quartic couplings $\left(\lambda_{i}, \lambda_{S}\right)$, Yukawa couplings $\left(Y_{i j}, h_{i \alpha}, y_{i}\right)$, gauge couplings $\left(g_{i}\right)$ should obey the perturbativity conditions:

$$
\begin{aligned}
\left|\lambda_{1,2,3,4,5,6,7}\right| & <4 \pi, & \left|\lambda_{S}\right|<4 \pi, \\
\left|Y_{u, d, e}, h_{i j}, y_{1,2}\right| & <\sqrt{4 \pi}, & \left|g_{s}, g, g^{\prime}\right|<\sqrt{4 \pi}
\end{aligned}
$$

\section{LEPTOGENESIS}

In this section, we discuss the details of a new way of generating lepton asymmetry at low scale in our model. Note that, similar to the minimal scotogenic model, here also there are two different ways of generating lepton asymmetry: out of equilibrium decay of the $N_{i}$ [21-30] or annihilation/scattering of dark sector particles $[69,70]$. In $[21,22]$, the authors considered a hierarchical right handed neutrino spectrum to show that successful leptogenesis from two body decay can occur at a scale as low as a few tens of $\mathrm{TeV}$. Leptogenesis with quasidegenerate right handed neutrinos in scotogenic model was discussed in [29,30]. In more recent works [24,25], successful leptogenesis was shown to be possible at a scale as low as $10 \mathrm{TeV}$ even with hierarchical right handed neutrinos while requiring to be in a weak washout regime predicting a vanishingly small lightest neutrino mass. If leptogenesis occurs only from two heavy neutrinos, then the scale of leptogenesis is pushed above the $\mathrm{TeV}$ scale by a few order of magnitudes [26]. This will correspond to our scenario if we do not have $\psi, S$ in our model. To summarize, it has been shown in the above mentioned works that the scale of leptogenesis in scotogenic model can be as low as a few $\mathrm{TeV}$ without requiring any resonance enhancement arising due to tiny mass splitting of right handed neutrinos. This significant improvement over the usual Davidson-Ibarra bound on the scale of leptogenesis $M_{N}>10^{9} \mathrm{GeV}$ for vanilla leptogenesis in type I seesaw framework [31] makes the scotogenic model a very attractive and testable framework for leptogenesis. We also note that high scale leptogenesis in scotogenic model was also studied recently by the authors of [71].

In addition to the usual $1 \rightarrow 2$ decay or $2 \rightarrow 2$ annihilations as sources of lepton asymmetry, we can also have $1 \rightarrow 3$ decay in our model. Such three body decay as a source of lepton asymmetry was discussed earlier by several authors [32-35] in the different contexts like radiative seesaw models, $\mathrm{R}$ parity violating supersymmetry and so on. Our present work is motivated two features namely, (i) dark matter particles assist in such three body decay processes and (ii) nonzero lepton asymmetry can be generated due to interference of three body decay diagrams with two different resummed propagators. We show that for our chosen regime of parameter space, such three body decay leptogenesis can be dominant over other possible sources of leptogenesis and the scale of leptogenesis can be lower than what was found by considering two body decay or annihilation processes discussed in earlier works.

In our model, we consider the three body decay of singlet fermion $\psi$ as the origin of $C P$ asymmetry through the process shown in Fig. 1. ${ }^{1}$ To prevent the two body decay of $\psi$ into $N_{i}, S$ we impose the kinematical constraint $m_{\psi}<M_{\alpha}+m_{S}$. The relevant decay processes that can generate Lepton asymmetry are $\psi \rightarrow S l \eta$ and $N_{1} \rightarrow \eta l$.

\footnotetext{
${ }^{1}$ In the absence of this process, the source of leptogenesis will be from decay of $N_{1,2}$ only and it was shown earlier that in such two right handed neutrino limit of scotogenic model, the scale of leptogenesis is pushed toward the higher side [26].
} 


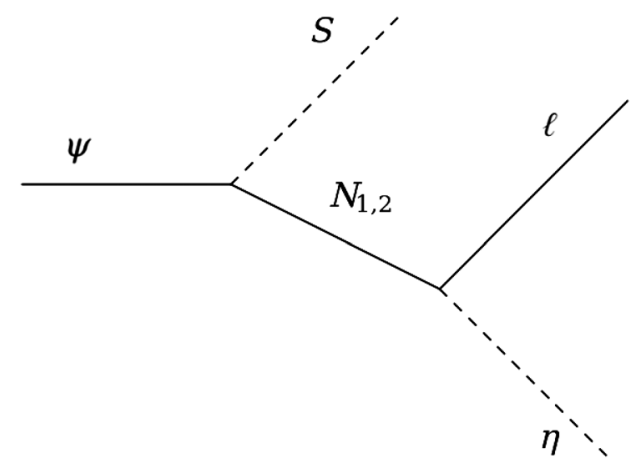

FIG. 1. Three body decay of singlet fermion $\psi$.

Although $N_{2}$ decay can also generate lepton asymmetry in principle, we consider the asymmetry generated by $N_{2}$ decay or any pre-existing asymmetry to be negligible due to strong washout effects mediated either by $N_{1}$ or $N_{2}$ themselves, to be discussed below. The corresponding $C P$ asymmetry parameters are defined as

$\epsilon_{\psi}=\frac{\Gamma_{\psi \rightarrow S l_{i} \eta}-\Gamma_{\psi \rightarrow S \bar{l}_{i} \eta^{*}}}{\Gamma_{\psi \rightarrow S l_{i} \eta}+\Gamma_{\psi \rightarrow S \bar{l}_{i} \eta^{*}}}, \quad \epsilon_{\left(N_{1}\right)_{i}}=\frac{\Gamma_{N_{1} \rightarrow l_{i} \eta}-\Gamma_{N_{1} \rightarrow \bar{l}_{i} \eta^{*}}}{\Gamma_{N_{1} \rightarrow l_{i} \eta}+\Gamma_{N_{1} \rightarrow \bar{l}_{i} \eta^{*}}}$

The details of these $C P$ asymmetry parameters are given in the Appendix A and B, respectively. Appendix A contains the details of one-loop calculation for resummed propagator in such three body decay, together with the usual tree plus one-loop level interference, giving rise to the same results. In the unflavored regime, we sum over lepton flavors $i$ to obtain the net $C P$ symmetries $\epsilon_{\psi}, \epsilon_{N_{1}}$ which will be used in the Boltzmann equations. Since we are considering sum over all lepton flavors, we are not going to show the flavor index explicitly in the following discussions.

Along with these two decay processes contributing to the creation of lepton asymmetry, there are washout processes too which tend to destroy the asymmetry created. The relevant washout processes in our model can be categorized as

(i) Inverse decays: $\ell \eta \rightarrow N_{1}, \ell \eta S \rightarrow \psi$

(ii) $\Delta L=1$ scatterings: $S \ell \rightarrow \psi \eta, \ell \eta \rightarrow \psi S, \psi \ell \rightarrow S \eta$, $\ell \eta \rightarrow N_{1}\left(W^{ \pm}, Z\right)$.

(iii) $\Delta L=2 \quad$ scatterings: $\quad \ell \ell \rightarrow N_{1} N_{1}, \quad l l \rightarrow \eta \eta$, $\eta \ell \rightarrow \eta^{*} \bar{\ell}$.

The Boltzmann equations relevant for leptogenesis in this model can be summarized as

$$
\begin{aligned}
\frac{d n_{\psi}}{d z}= & -D_{\psi}\left(n_{\psi}-n_{\psi}^{\mathrm{eq}}\right)+D_{N_{1} \rightarrow \psi S}\left(n_{N_{1}}-n_{N_{1}}^{e q}\right) \\
& -W_{I D_{N_{1} \rightarrow \psi S}} n_{\psi}-\frac{s}{H(z) z}\left[\left(n_{\psi} n_{\eta}-n_{\psi}^{\mathrm{eq}} n_{\eta}^{\mathrm{eq}}\right)\langle\sigma v\rangle_{\psi \eta \rightarrow S l}\right. \\
& \left.+\left[n_{\psi} n_{S}-n_{\psi}^{\mathrm{eq}} n_{S}^{\mathrm{eq}}\right]\langle\sigma v\rangle_{\psi S \rightarrow l \eta}\right],
\end{aligned}
$$

$$
\begin{aligned}
\frac{d n_{N_{1}}}{d z}= & -D_{N_{1}}\left(n_{N_{1}}-n_{N_{1}}^{\mathrm{eq}}\right)-D_{N_{1} \rightarrow \psi S}\left(n_{N_{1}}-n_{N_{1}}^{\mathrm{eq}}\right) \\
& -\frac{s}{H(z) z}\left[\left(n_{N_{1}}^{2}-\left(n_{N_{1}}^{\mathrm{eq}}\right)^{2}\right)\langle\sigma v\rangle_{N_{1} N_{1} \rightarrow l l}\right. \\
& \left.+\left[n_{N_{1}} n_{\mathrm{SM}}-n_{N_{1}}^{\mathrm{eq}} n_{\mathrm{SM}}^{\mathrm{eq}}\right]\langle\sigma v\rangle_{\eta l \rightarrow N_{1}\left(W^{ \pm}, Z\right)}\right] \\
\frac{d n_{B-L}}{d z}= & -\epsilon_{\psi} D_{\psi}\left(n_{\psi}-n_{\psi}^{\mathrm{eq}}\right)-\epsilon_{N_{1}} D_{N_{1}}\left(n_{N_{1}}-n_{N_{1}}^{\mathrm{eq}}\right) \\
& -\left(W_{N_{1}}+W_{\psi}\right) n_{B-L} \\
& -\frac{s}{H(z) z}\left[\Gamma_{S l \rightarrow \psi \eta}+\Gamma_{l \eta \rightarrow \psi S}+\Gamma_{l l \rightarrow \eta \eta}+\Gamma_{l l \rightarrow N_{1} N_{1}}\right. \\
& \left.+\Gamma_{l \eta \rightarrow\left(N_{1} W^{ \pm}, Z\right)}+\Gamma_{\eta l \rightarrow \eta^{*} l}\right] n_{B-L} .
\end{aligned}
$$

In the above equations $z=\frac{m_{\psi}}{T}$ and $n_{f}^{\mathrm{eq}}=\frac{z^{2}}{2} \kappa_{2}(z)$ is the equilibrium number density of $f \equiv N_{1}, \psi$ (with $\kappa_{i}(z)$ being the modified Bessel function of $i$ th kind). The quantity $D_{f}$ on the right-hand side of the above equations is

$\begin{aligned} D_{N_{1}} & =K_{N_{1}} z\left(\frac{M_{1}}{m_{\psi}}\right) \frac{\kappa_{1}\left[z\left(\frac{M_{1}}{m_{\psi}}\right)\right]}{\kappa_{2}\left[z\left(\frac{M_{1}}{m_{\psi}}\right)\right]}, \\ D_{\psi} & =K_{\psi} z \frac{\kappa_{1}(z)}{\kappa_{2}(z)}, \quad D_{N_{1} \rightarrow \psi S}=K_{N_{1} \rightarrow \psi S} z\left(\frac{M_{1}}{m_{\psi}}\right) \frac{\kappa_{1}\left[z\left(\frac{M_{1}}{m_{\psi}}\right)\right]}{\kappa_{2}\left[z\left(\frac{M_{1}}{m_{\psi}}\right)\right]} .\end{aligned}$

Here, the decay parameters are defined as

$K_{N_{1}}=\frac{\Gamma_{N_{1} \rightarrow l \eta}}{H\left(m_{\psi}\right)}, \quad K_{\psi}=\frac{\Gamma_{\psi \rightarrow S l \eta}}{H\left(m_{\psi}\right)}, \quad K_{N_{1} \rightarrow \psi S}=\frac{\Gamma_{N_{1} \rightarrow \psi S}}{H\left(m_{\psi}\right)}$

with $\Gamma_{f}$ is the partial decay width of particle $f$ for the specified decay process, $H$ is the Hubble parameter. Since leptogenesis is a high scale phenomena and occurs in the radiation dominated phase of the universe, the Hubble parameter can be expressed in terms of the temperature $T$ as follows

$$
H=\sqrt{\frac{8 \pi^{3} g_{*}}{90}} \frac{T^{2}}{M_{\mathrm{Pl}}}=H(z=1) \frac{1}{z^{2}}
$$

where $g_{*}$ is the effective number of relativistic degrees of freedom and $M_{\mathrm{Pl}} \simeq 1.22 \times 10^{19} \mathrm{GeV}$ is the Planck mass. The washout terms are given as

$$
\begin{aligned}
W_{N_{1}} & =\frac{1}{4} z^{3}\left(\frac{M_{1}}{m_{\psi}}\right)^{2} K_{N_{1}} \kappa_{1}\left[z \frac{M_{1}}{m_{\psi}}\right], \quad W_{\psi}=\frac{1}{4} z^{3} K_{\psi} \kappa_{1}(z), \\
W_{N_{1} \rightarrow \psi S} & =\frac{1}{4} z \frac{\kappa_{1}\left[z \frac{M_{1}}{m_{\psi}}\right]}{\kappa_{2}\left[z \frac{M_{1}}{m_{\psi}}\right]} K_{N_{1} \rightarrow \psi S} \frac{n_{N_{1}}^{e q}}{n_{\psi}^{e q}}
\end{aligned}
$$

The decay process $N_{1} \rightarrow \psi S$ does not contribute to the $C P$ asymmetry but can affect the abundance of $\psi, N_{1}$ as can 
be seen from the Boltzmann equations written above. The decay width for the decay $N_{1} \rightarrow \psi S$ is given by

$$
\begin{aligned}
\Gamma_{N_{1} \rightarrow \psi S}= & \frac{1}{16 \pi M_{1}^{3}} \sqrt{M_{1}^{4}+m_{\psi}^{4}+m_{S}^{4}-2 M_{1}^{2} m_{\psi}^{2}-2 m_{\psi}^{2} m_{S}^{2}} \\
& \times\left\{\left(M_{1}^{2}+m_{\psi}^{2}-m_{S}^{2}\right)\left|y_{1}\right|^{2}-2 \operatorname{Re}\left[y_{1}^{2}\right] m_{\psi} M_{1}\right\} .
\end{aligned}
$$

As mentioned earlier, we use the Casas-Ibarra parametrization to rewrite the Yukawa coupling $h_{i j}$ in terms of light neutrino parameters. Also, in the case of two right

$$
h=\left(\begin{array}{l}
\sqrt{m_{2}} \sqrt{\Lambda_{1}} \cos (z) U_{12}+\sqrt{m_{3}} \sqrt{\Lambda_{1}} \sin (z) U_{13} \\
\sqrt{m_{2}} \sqrt{\Lambda_{1}} \cos (z) U_{22}+\sqrt{m_{3}} \sqrt{\Lambda_{1}} \sin (z) U_{23} \\
\sqrt{m_{2}} \sqrt{\Lambda_{1}} \cos (z) U_{32}+\sqrt{m_{3}} \sqrt{\Lambda_{1}} \sin (z) U_{33}
\end{array}\right.
$$

with $U_{i j}$ being the elements of the PMNS mixing matrix mentioned earlier. The other Yukawa coupling which affects lepton asymmetry namely, $y_{i}$ is not related to the origin of light neutrino mass and hence we keep it as a free parameter. The choice of this Yukawa coupling affect both leptogenesis and dark matter as we discuss in upcoming sections.

After obtaining the numerical solutions of the above Boltzmann equations (33), (34) and (35), we convert the final $B-L$ asymmetry $n_{B-L}^{f}$ just before electroweak sphaleron freeze-out into the observed baryon to photon ratio by the standard formula

$$
\eta_{B}=\frac{3}{4} \frac{g_{*}^{0}}{g_{*}} a_{\mathrm{sph}} n_{B-L}^{f} \simeq 9.2 \times 10^{-3} n_{B-L}^{f},
$$

where $a_{\text {sph }}=\frac{8}{23}$ is the sphaleron conversion factor (taking into account two Higgs doublets). We take the effective relativistic degrees of freedom to be $g_{*}=111.75$, slightly higher than that of the SM at such temperatures as we are including the contribution of the inert doublet as well as the scalar singlet too. The heavy singlet fermions $N_{1,2}, \psi$ do not contribute as they have already decoupled from the bath by this epoch. In the above expression $g_{*}^{0}=\frac{43}{11}$ is the effective relativistic degrees of freedom at the recombination epoch.

\section{DARK MATTER}

As mentioned earlier, our model has two DM candidates both of which are stable due to the unbroken $Z_{2} \times Z_{2}^{\prime}$ symmetry. Although a two component DM was not part of the original motivation, it emerged naturally due to the chosen charge assignments of different particles namely, $\eta$, $S, \psi, N_{i}$ under $Z_{2} \times Z_{2}^{\prime}$ symmetry. In fact, the introduction handed neutrinos taking part in generating light neutrino masses in our model, the complex orthogonal matrix $R$ is a function of only one rotation parameter $z=z_{R}+i z_{I}, z_{R} \in$ $[0,2 \pi], z_{I} \in \mathbb{R}[50,52]$. Our choice of $R$ matrix is

$$
R=\left(\begin{array}{ccc}
0 & \cos \left(z_{R}+i z_{I}\right) & \sin \left(z_{R}+i z_{I}\right) \\
0 & -\sin \left(z_{R}+i z_{I}\right) & \cos \left(z_{R}+i z_{I}\right)
\end{array}\right)
$$

Then the Yukawa matrix for normal ordering of light neutrino masses can then be explicitly written as

$$
\left.\begin{array}{l}
-\sqrt{m_{2}} \sqrt{\Lambda_{2}} \sin (z) U_{12}+\sqrt{m_{3}} \sqrt{\Lambda_{2}} \cos (z) U_{13} \\
-\sqrt{m_{2}} \sqrt{\Lambda_{2}} \sin (z) U_{22}+\sqrt{m_{3}} \sqrt{\Lambda_{2}} \cos (z) U_{23} \\
-\sqrt{m_{2}} \sqrt{\Lambda_{2}} \sin (z) U_{32}+\sqrt{m_{3}} \sqrt{\Lambda_{2}} \cos (z) U_{33} .
\end{array}\right)
$$

of the second $Z_{2}$ symmetry, necessary to forbid direct coupling of $\psi$ with SM leptons, has given rise to the second DM component in the model. A very recent study on such two component DM with scalar doublet and scalar singlet can be found in [72]. For some earlier works on multicomponent dark matter, please refer to [45,46,56,70,73-89,89-104] and references therein.

Relic abundance of two component DM in our model $\eta_{R}, S$ can be found by numerically solving the corresponding Boltzmann equations. Let $n_{1}=n_{\eta_{R}}$ and $n_{2}=n_{S}$ be the total number densities of two dark matter candidates, respectively. The two coupled Boltzmann equations in terms of $n_{2}$ and $n_{1}$ are given below [45],

$$
\begin{aligned}
\frac{d n_{1}}{d t}+3 n_{1} H= & -\left\langle\sigma \mathrm{v}_{\eta_{R} \eta_{R} \rightarrow X \bar{X}}\right\rangle\left(n_{1}^{2}-\left(n_{1}^{\mathrm{eq}}\right)^{2}\right) \\
& -\left\langle\sigma \mathrm{v}_{\eta_{R} \eta_{R} \rightarrow S S}\right\rangle\left(n_{1}^{2}-\frac{\left(n_{1}^{\mathrm{eq}}\right)^{2}}{\left(n_{2}^{\mathrm{eq}}\right)^{2}} n_{2}^{2}\right), \\
\frac{d n_{2}}{d t}+3 n_{2} H= & -\left\langle\sigma \mathrm{v}_{S S \rightarrow X \bar{X}}\right\rangle\left(n_{2}^{2}-\left(n_{2}^{\mathrm{eq}}\right)^{2}\right) \\
& +\left\langle\sigma \mathrm{v}_{\eta_{R} \eta_{R} \rightarrow S S}\right\rangle\left(n_{1}^{2}-\frac{\left(n_{1}^{\mathrm{eq}}\right)^{2}}{\left(n_{2}^{\mathrm{eq}}\right)^{2}} n_{2}^{2}\right),
\end{aligned}
$$

where, $n_{i}^{\mathrm{eq}}$ is the equilibrium number density of dark matter species $i$ and $H$ denotes the Hubble parameter, defined earlier. In the annihilation processes, $\mathrm{X}$ denotes all particles where DM can annihilate into. In the above equations, $\langle\sigma v\rangle$ is the thermally averaged annihilation cross section, given by [105]

$$
\begin{aligned}
\langle\sigma v\rangle_{\mathrm{DMDM} \rightarrow \mathrm{X} \overline{\mathrm{X}}}= & \frac{1}{8 m_{\mathrm{DM}}^{4} T \kappa_{2}^{2}\left(\frac{m_{\mathrm{DM}}}{T}\right)} \int_{4 m_{\mathrm{DM}}^{2}}^{\infty} \sigma\left(s-4 m_{\mathrm{DM}}^{2}\right) \\
& \times \sqrt{s} \kappa_{1}\left(\frac{\sqrt{s}}{T}\right) d s,
\end{aligned}
$$




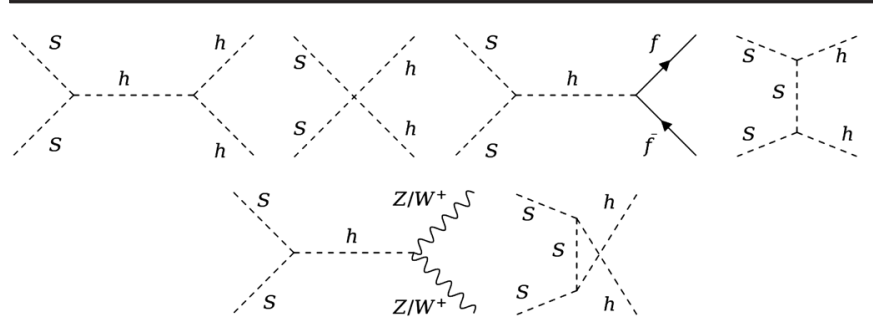

FIG. 2. Feynman diagrams for relevant annihilation processes for singlet scalar DM.

where $\kappa_{i}(x)$ 's are modified Bessel functions of order $i$ mentioned before. The annihilation processes of scalar singlet scalar doublet are shown in Fig. 2 and 3 respectively. While for scalar singlet DM alone, there is no coannihilation processes, scalar doublet dark matter in scotogenic model can have several coannihilation processes, either with the heavier components of the doublet or fermions as shown in Fig. 3. Such coannihilation effects within the framework of inert doublet model as well as scotogenic model have already been studied in details by several authors $[21,58,106-119]$. In the presence of coannihilations, one follows the recipe given by [120] to calculate the relic abundance. Since scalar singlet DM has just one component, there is no such coannihilations present. Similar to the inert doublet dark matter model, scalar singlet dark matter has also been studied extensively by several authors [121-125].

The second terms on the right-hand side of the above Boltzmann equations specifically consider the conversions between two DM candidates $\eta_{R}, S$ while assuming the former to be the heavier DM component. Such a conversion can occur either directly due to the $\lambda_{6}$ coupling of the scalar potential given in equation (3) or via SM
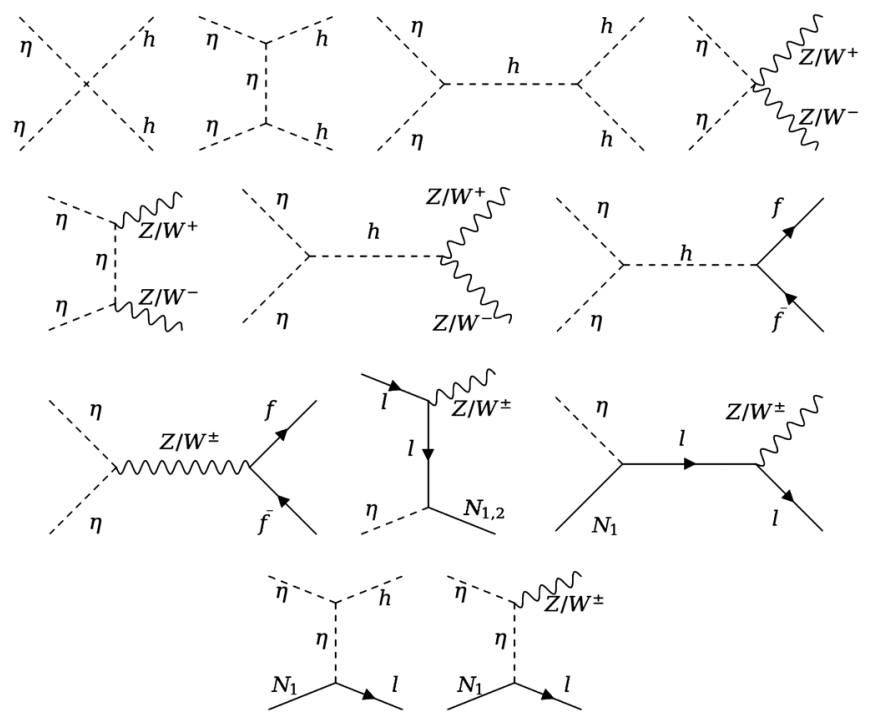

FIG. 3. Feynman diagrams of all the relevant processes for scalar doublet dark matter in the scotogenic model. Here DM is chosen to be the real scalar component of the doublet.

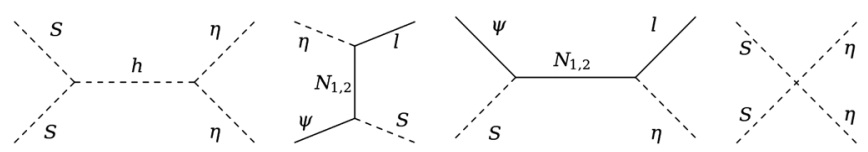

FIG. 4. Feynman diagrams of all the relevant processes determining the DM relic density which emerged due to the extension of the scotogenic model.

Higgs portal interactions. These conversion processes are shown in Fig. 4. There can be another conversion process due to the interactions shown in the Feynman diagram of Fig. 1. This can occur due to coannihilation processes, not shown in the above Boltzmann equations. In our model, however, singlet scalar DM can, in principle, coannihilate with other particles involved in the same Feynman diagram of Fig. 1. Since the two DM candidates are stabilized by two separate $Z_{2}$ symmetries, their coannihilation can only lead to $\psi$ which is odd under both the $Z_{2}$ symmetries. Alternatively, one of the DM can also coannihilate with $\psi$ and convert into the other DM. These processes are shown in Fig. 4. Since we consider $\psi$ to be heavier than both the DM candidates, we do not show it in the final states.

In order to cover all the features of annihilations, coannihilations as well as conversions, we use MICROMEGAS [126] to calculate the relic abundance of two component DM in our model. The model information has been supplied to MICROMEGAS using FEYNRULES [127] while all the relevant annihilation and coannihilation cross sections of dark matter number changing processes required to solve the coupled equations are calculated using CALCHEP [128]. While singlet scalar DM annihilates either through four point scalar interactions or SM Higgs mediated processes, the scalar doublet DM can annihilate (coannihilate) via Higgs as well as electroweak gauge boson portals apart from the four point interactions with Higgs as well as gauge bosons. Additionally, the conversion coupling $\lambda_{6}$ as well as Yukawa coupling $y_{i}$ can play significant role in individual as well as total DM relic densities.

Just like the SM Higgs boson mediates DM annihilation into SM particles, similarly, it can also mediate spin independent DM-nucleon scatterings. Different ongoing experiments like Xenon1T [129,130], LUX [131], PandaX-II $[132,133]$ are trying to detect the DM in the labbased experiments and give a strong upper bound on the spin-independent (SI) direct detection (DD) cross-section as a function of DM mass. We have extracted the SI elastic scattering cross-section for both the DM candidates from MICROMEGAS. DD analysis for two-component DM is slightly different from the single component scenario. To compare the result of our model with Xenon1T bound, we have multiplied the elastic scattering cross section by the relative number density of each DM candidate and used the following conditions 

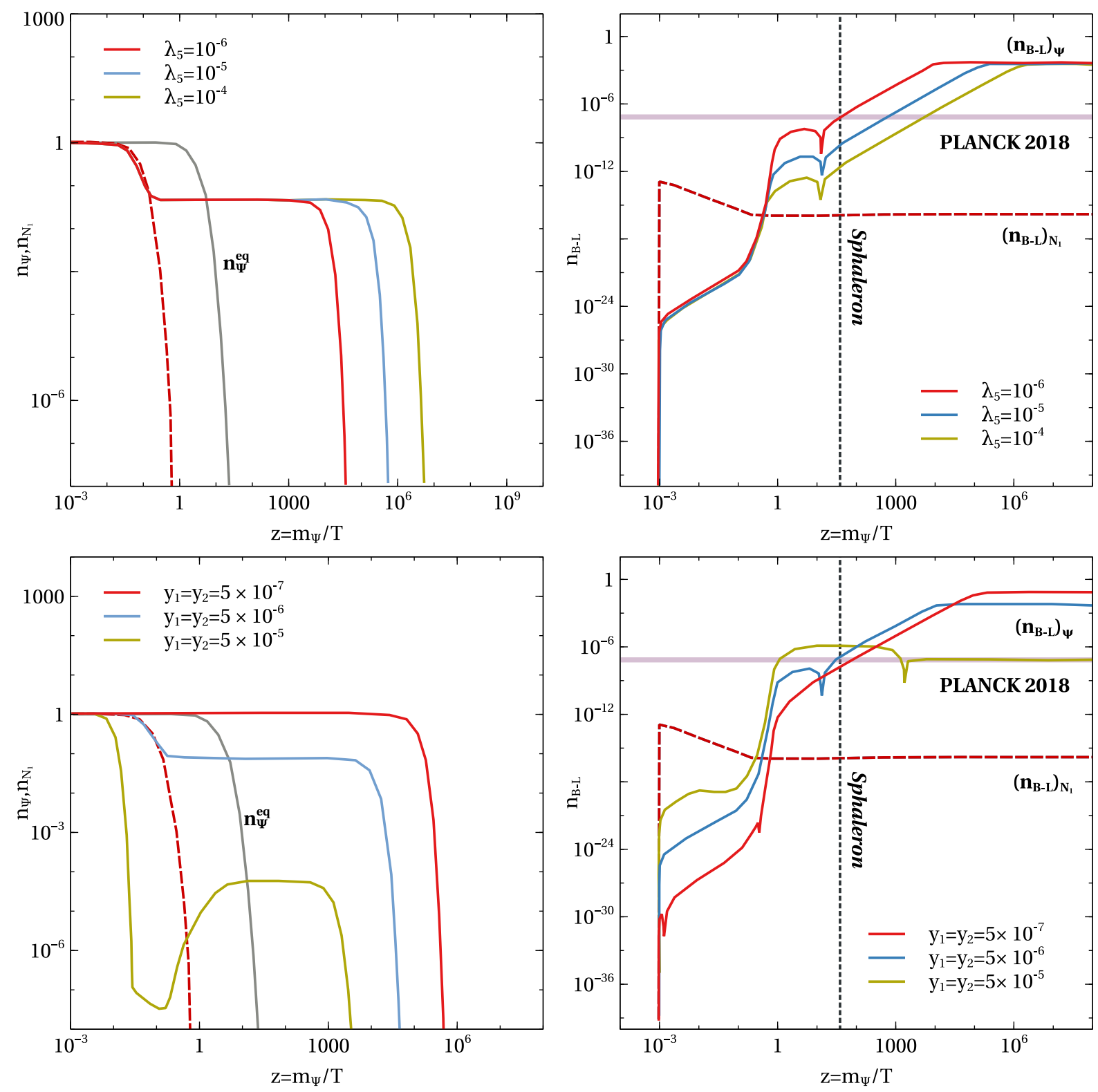

FIG. 5. Evolution of comoving number densities of $\psi$ and $N_{1}$ (left panel) and $B-L$ (right panel) with $z=\frac{m_{\psi}}{T}$ for different values of $\lambda_{5}$ (upper panel), Yukawa couplings $y_{1,2}$ (lower panel). The other parameters are set at benchmark values: $M_{1}=2 \times 10^{5} \mathrm{GeV}$, $M_{2}=2 \times 10^{6} \mathrm{GeV}, m_{\eta}=100 \mathrm{GeV}, m_{S}=500 \mathrm{GeV}, m_{\psi}=5 \mathrm{TeV}$ and $y_{1}=y_{2}=5 \times 10^{-6}$ (upper panel) and $\lambda_{5}=10^{-6}$ (lower panel).

$$
\begin{aligned}
& \sigma_{1}^{\text {eff }}=\frac{\mathrm{n}_{1}}{\mathrm{n}_{1}+\mathrm{n}_{2}} \sigma_{1}^{\mathrm{SI}} \leq \sigma_{\text {Xenon } 1 \mathrm{~T}} \\
& \sigma_{2}^{\text {eff }}=\frac{\mathrm{n}_{2}}{\mathrm{n}_{1}+\mathrm{n}_{2}} \sigma_{2}^{\mathrm{SI}} \leq \sigma_{\text {Xenon } 1 \mathrm{~T}}
\end{aligned}
$$

Further details related to the direct detection of multicomponent DM can be found in $[134,135]$.

\section{RESULTS AND DISCUSSION}

In this section, we discuss our numerical results for leptogenesis as well as dark matter separately.

\section{A. Leptogenesis}

To calculate the lepton asymmetry, we first solve the coupled Boltzmann equations (33), (34), and (35) numerically to estimate the final B-L asymmetry. We considered two possible ranges for $N_{1}$ mass $M_{1}$.

In the first case we have chosen a benchmark as $M_{1}=$ $2 \times 10^{5} \mathrm{GeV}$ and in the other case we choose $M_{1}=2 \times$ $10^{7} \mathrm{GeV}$ while keeping $M_{2}=10 M_{1}$ and other parameters fixed for both the cases. Using the first choice of benchmark values for $M_{1}$ and $M_{2}$, in Fig. 5, the evolution of the comoving number densities of $\psi, N_{1}$ and $B-L$ are shown 

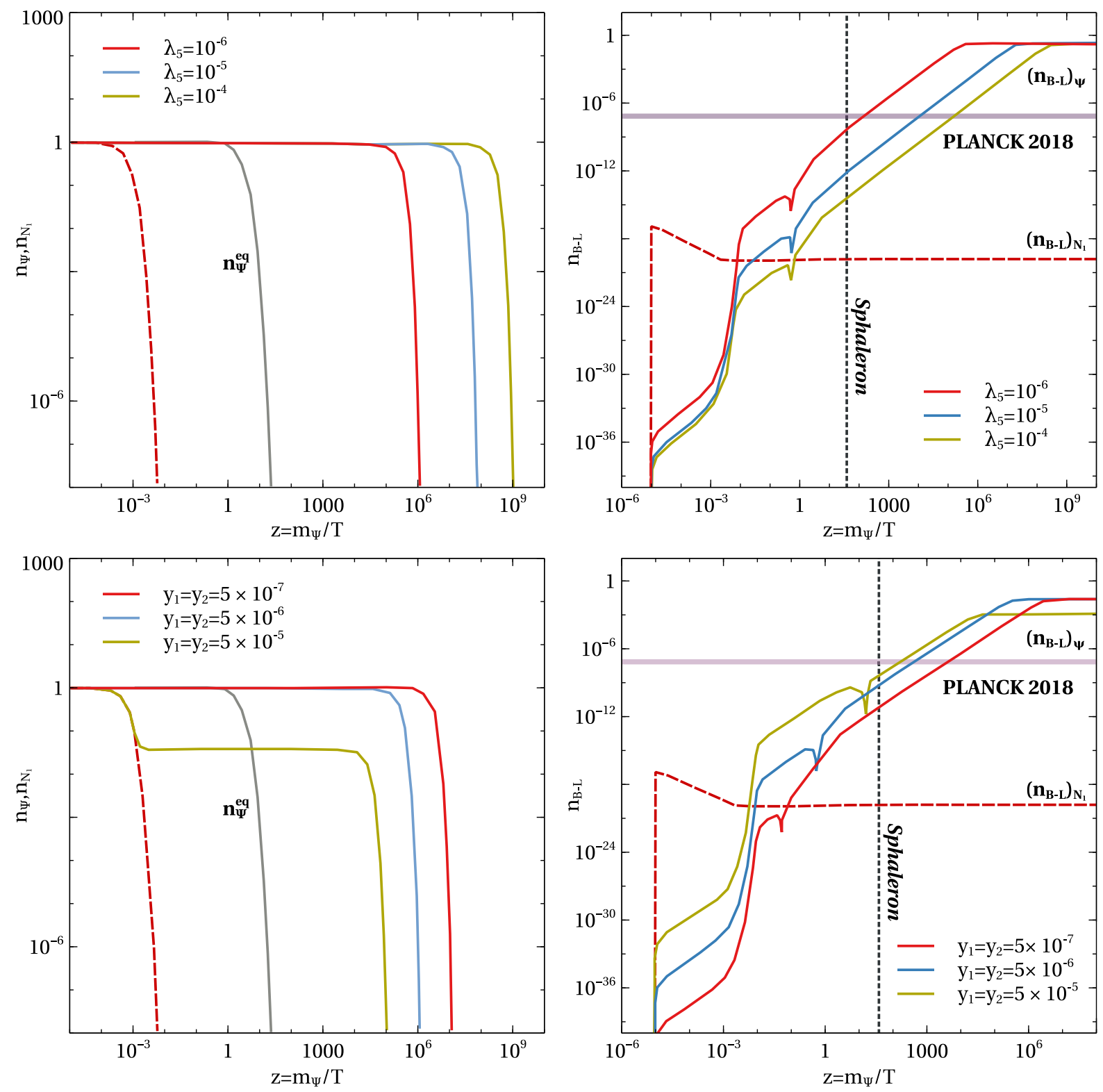

FIG. 6. Evolution of comoving number densities of $\psi$ and $N_{1}$ (left panel) and $B-L$ (right panel) with $z=\frac{m_{\psi}}{T}$ for different values of $\lambda_{5}$ (upper panel), Yukawa couplings $y_{1,2}$ (lower panel). The other parameters are set at benchmark values: $M_{1}=2 \times 10^{7} \mathrm{GeV}$, $M_{2}=2 \times 10^{8} \mathrm{GeV}, \quad m_{\eta}=100 \mathrm{GeV}, \quad m_{S}=500 \mathrm{GeV}, \quad m_{\psi}=5 \mathrm{TeV}$ and $y_{1}=y_{2}=5 \times 10^{-6}$ (upper panel) and $\lambda_{5}=10^{-6}$ (lower panel).

with $z=\frac{m_{\psi}}{T}$ for different values of $\lambda_{5}$ and $y_{1,2}$. Similarly in Fig. 6, the second benchmark for RHN masses is chosen and the evolution of the comoving number densities of $\psi$, $N_{1}$ and $B-L$ are shown with $z=\frac{m_{\psi}}{T}$ for different values of $\lambda_{5}$ and $y_{1,2}$. The parameter $\lambda_{5}$ decides the strength of Dirac Yukawa coupling of neutrinos via Casas-Ibarra parametrization as (13) discussed earlier.

In the three body decay width $\Gamma_{\psi \rightarrow l S \eta}$, apart from Dirac Yukawa couplings of active neutrinos, we also have other Yukawa couplings $y_{1,2}$ which can affect leptogenesis, without affecting neutrino mass. In this section we discuss the effect of these two types of Yukawa couplings on the asymmetry. In the upper left panel of Fig. 5 we show the evolution of the comoving number densities of $\psi$ (solid lines) and $N_{1}$ (dashed lines) for different benchmark values of $\lambda_{5}$. In the upper right panel of Fig. 5, we show the evolution of $B-L$ for different $\lambda_{5}$. We have taken both $\psi$ and $N_{1}$ to be in equilibrium at very high temperatures and numerically solved the coupled Boltzmann equations upto a temperature when the asymmetry gets saturated. From the evolution of the comoving number densities of $\psi$ and $N_{1}$ we can see that initially both the particles follow their equilibrium number densities but soon after the $\psi$ 
abundance deviates from its equilibrium abundance while the $N_{1}$ abundance remains very close to its equilibrium number density and therefore vanishes shortly after it becomes nonrelativistic. It is mainly because $N_{1}$ has very strong two-body decays $\left(N_{1} \rightarrow \ln\right.$ and $\left.N_{1} \rightarrow \psi S\right)$ and corresponding inverse decays and therefore its abundance remains very close to its equilibrium abundance. On the other hand, $\psi$ has relatively feeble three-body decay $\psi \rightarrow S \eta l$ and a strong inverse decay $\psi S \rightarrow N_{1}$. Because of the strong two-body inverse decay of $\psi$ the abundance of $\psi$ decreases sharply. This inverse decay stops when the temperature drops to a value such that the process $\psi S \rightarrow N_{1}$ becomes kinematically forbidden. After this point the $\psi$ abundance gets saturated and goes out of equilibrium before finally decaying through the three-body decay at a low temperature. The effect of this strong inverse decay $\left(\psi S \rightarrow N_{1}\right)$ can also be seen in the asymmetry evolution shown on the right panel plot. The solid lines represent the asymmetry generated from the $\psi$ decay and the dashed line represents the asymmetry generated from the two-body decay of $N_{1}$. From asymmetry plots on right panel of Fig. 5, it can be seen that asymmetry generated from the $N_{1}$ decay is very less compared to the one generated from the threebody decay of $\psi$, which is expected as $N_{1}$ remains very close to its equilibrium abundance. Another important point is the asymmetry generated from $N_{1}$ gets saturated at a very high temperature as $N_{1}$ abundance vanishes when it becomes nonrelativistic. However, the asymmetry generated from the $\psi$ decay keep evolving up to a very low temperature because of the small decay width of $\psi$. Because of the small decay parameter for the decay $\psi \rightarrow S \eta l$, the inverse decay rate is also very small and we are always in a weak washout regime $\left(K_{\psi} \ll 1\right)$. However, the asymmetry generation from the two-body decay of $N_{1}$ is always is in strong washout regime as we have only two RHNs (for two RHNs scenarios, $K_{N_{1}}>1$ ) $[24,27]$. Because of this, the resultant asymmetry is mainly determined by the three-body decay.

In the upper panel plot of Fig. 5 it is observed that abundance of $\psi$ become less than its equilibrium abundance because of the inverse decay $\psi \rightarrow N_{1} S$ (while $N_{1}$ has strong two-body decays into both $\psi S$ and $\eta l$ final states) and therefore the asymmetry generated from the $\psi$ decay remains suppressed initially. However, when the inverse decay stops, the $\psi$ abundance saturates and finally the asymmetry becomes overabundant before its decay is complete. When the $\psi$ becomes more compared to its equilibrium abundance then the $B-L$ asymmetry starts rising steadily, as can be seen by comparing left and right panel plots of Fig. 5. In upper left panel plot of Fig. 5 we can see that the decay of $\psi$ happens earlier for smaller value of $\lambda_{5}$, which is expected as smaller value of $\lambda_{5}$ lead to larger Dirac Yukawa couplings. For the same reason the saturation of asymmetry happens earlier for smaller value values of $\lambda_{5}$, as seen from upper right panel plot. The asymmetry generated up to the sphaleron epoch $\left(T_{\text {Sphaleron }} \simeq 131 \mathrm{GeV}\right.$, shown by vertical dashed line $)$ is important as the asymmetry generated after the sphaleron freeze-out temperature can not be converted into a baryon asymmetry. It can be seen from upper right panel plot of Fig. 5 that for smaller $\lambda_{5}$ the asymmetry generated upto sphaleron temperature is more compared to the ones for larger $\lambda_{5}$. In the lower left panel plot of Fig. 5 we observe that for larger values of the Yukawa couplings $y_{1,2}$, the effect of the inverse decay $\psi S \rightarrow N_{1}$ is more. For larger Yukawa coupling $y_{1,2}$ the $\psi$ abundance decreases sharply because of the very strong inverse decay $\psi S \rightarrow N_{1}$ and at later epochs also when its abundance is more compared to equilibrium abundance, smaller Yukawa leads to larger abundance as expected. For the same reason, the $B-L$ asymmetry also increases more sharply for larger Yukawa couplings leading to larger asymmetry at the epoch of sphaleron freeze-out, as seen from the lower right panel plot of Fig. 5. It should be noted that, we are showing only the absolute value of $B-L$ asymmetry on the right panel plots; in reality, the points toward the left of the dip in solid lines correspond to negative asymmetry. Clearly, for large Yukawa $y_{1,2}$, the asymmetry remains negative even at the sphaleron epoch, as seen from lower right panel plot. Therefore, we cannot make Yukawa coupling $y_{1,2}$ arbitrarily large to get more asymmetry at the epoch of shpaleron decoupling.

Similarly, in Fig. 6 we have shown the evolution $\psi, N_{1}$ and $B-L$ number densities with $z=m_{\psi} / T$ for different values of $\lambda_{5}$ (upper panel) and $y_{1,2}$ (lower panel) but with heavier mediator masses namely, $M_{1}=2 \times 10^{7} \mathrm{GeV}$ and $M_{2}=10 M_{1}$, keeping other parameters fixed as in Fig. 5. It can be seen that for the same value of $m_{\psi}, \lambda_{5}, m_{\eta}, m_{S}$, and $y_{1,2}$ the effect of the inverse decay $\psi S \rightarrow N_{1}$ is much less in this case. This is expected as for larger value of $N_{1}$ will make the inverse decay $\psi S \rightarrow N_{1}$ inefficient even at very high temperatures. The variation of the $B-L$ asymmetry with $\lambda_{5}$ and $y_{1,2}$ can be understood in a way similar to the Fig. 5 discussed earlier. Also, comparing Figs. 5 and 6 we can see that for the same set of parameters the decay of $\psi$ and generation of asymmetry occur slowly making the asymmetry less in Fig. 6 than in 5 at the epoch of sphaleron decoupling. This is expected as larger $N_{1,2}$ masses make the three-body decay width of $\psi$ smaller due to propagator suppression.

Finally, we perform a numerical scan to find the relevant parameter space in $m_{\psi}-\lambda_{5}$ plane that can give rise to the observed baryon asymmetry for both $M_{1}=2 \times 10^{5} \mathrm{GeV}$ and $M_{1}=2 \times 10^{7} \mathrm{GeV}$. While varying these parameters, we keep the masses of other relevant particles to be fixed at $m_{S}=500 \mathrm{GeV}, m_{\eta}=100 \mathrm{GeV}$. The parameter space in $m_{\psi}-\lambda_{5}$ plane for benchmark choices of $y_{1,2}$ is shown in Fig. 7 for $M_{1}=M_{2} / 10=2 \times 10^{5} \mathrm{GeV}$ (left panel) and for $M_{1}=M_{2} / 10=2 \times 10^{7} \mathrm{GeV}$ (right panel). In Fig. 7 we can see that for a benchmark value of $y_{1,2}$ the mass required of $\psi$ become large for larger values of $\lambda_{5}$, which is 

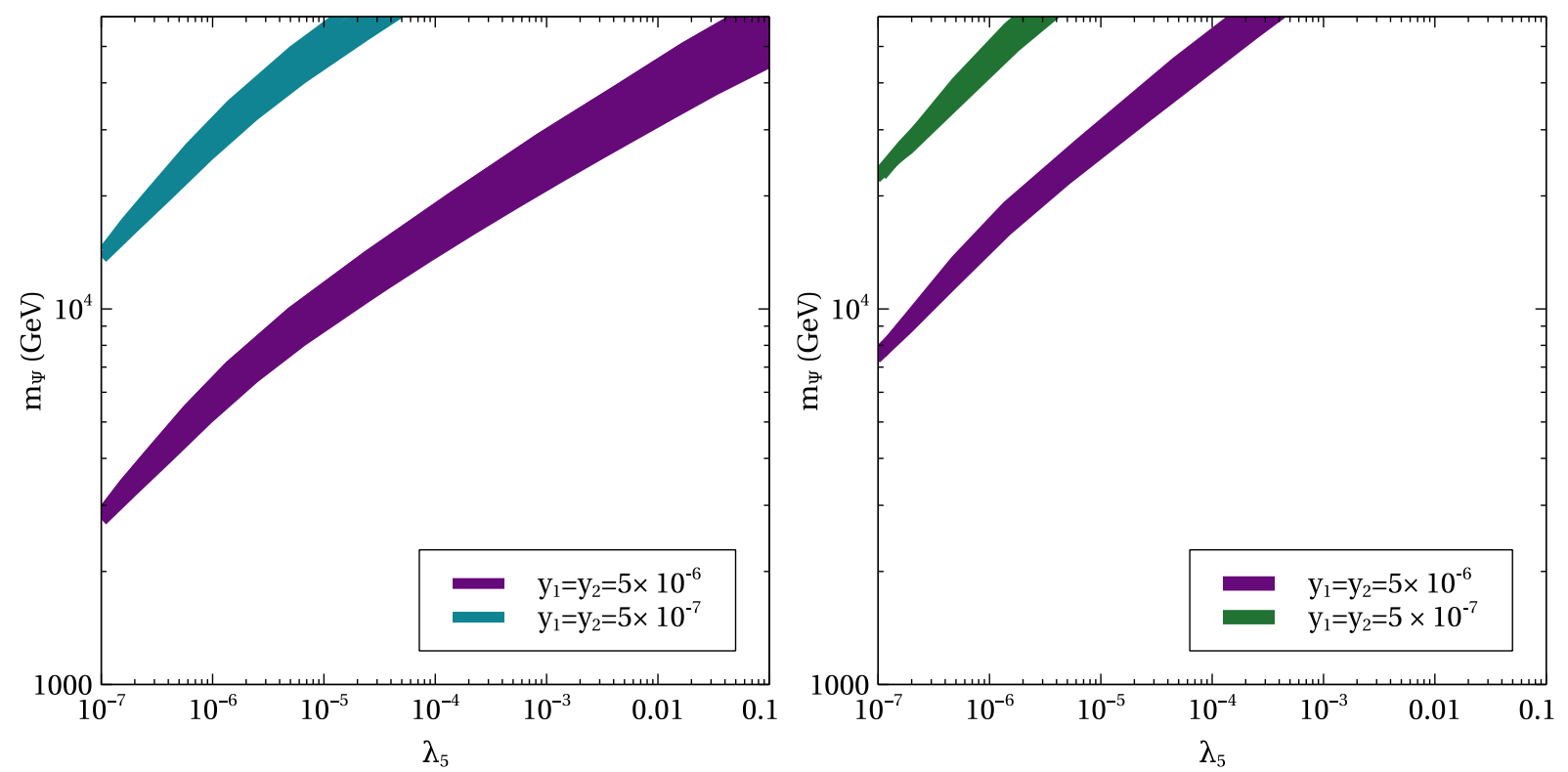

FIG. 7. The variation of $m_{\psi}$ with $\lambda_{5}$, required to satisfy the observed asymmetry. For the left panel the $M_{1}=2 \times 10^{5} \mathrm{GeV}, M_{2}=$ $2 \times 10^{6} \mathrm{GeV}$ and for the right panel $M_{1}=2 \times 10^{7} \mathrm{GeV}, M_{2}=2 \times 10^{8} \mathrm{GeV}$. The other parameters are set at $m_{\eta}=10 \mathrm{GeV}$, $m_{S}=500 \mathrm{GeV}$.

expected as larger values of $\lambda_{5}$ make the Dirac Yukawa couplings $h_{i \alpha}$ smaller making the three body decay width of $\psi$ smaller. For similar reason, for a particular value of $\lambda_{5}$, the required mass of $\psi$ for a small Yukawa coupling $y_{1,2}$ is more compared to that for larger Yukawa coupling. However, as mentioned earlier we cannot take arbitrarily large values of the new Yukawa couplings $y_{1,2}$ to lower the scale of leptogenesis. As discussed earlier, beyond a certain value of $y_{1,2}$ the inverse decay $\psi S \rightarrow N_{1}$ will become so dominant that the asymmetry become negative at the time of sphaleron. We found that for a $\mathrm{TeV}$ scale leptogenesis with moderately high $M_{1,2}$ it is safe to take $y_{1,2} \leq 10^{-5} \mathrm{such}$ that the asymmetry become positive at the sphaleron freezeout temperature. From Fig. 7 we can conclude that successful $\mathrm{TeV}$ scale leptogenesis is possible dominantly from the three body decay for appropriate choice of the model parameters. Also we can see that the scale of leptogenesis is slightly higher in the right panel plot of Fig. 7 compared to the left plot of Fig. 7. This is because with the increases in $M_{1,2}$ the three body decay width encounters propagator suppression as discussed earlier.

\section{B. Flavor effects on leptogenesis}

It should be noted that while discussing leptogenesis in the above sections, we did not consider the effects of lepton flavors. Since we are considering leptogenesis at low scale, lepton flavor effects may play nontrivial roles as pointed out by several earlier works on flavored leptogenesis [136-139], also summarized in a recent review article [140]. Adopting the notations of [139], the Boltzmann equations for flavored leptogenesis can be written as

$$
\begin{gathered}
\frac{d n_{\psi}}{d z}=-D_{\psi}\left(n_{\psi}-n_{\psi}^{\mathrm{eq}}\right)+D_{N_{1} \rightarrow \psi S}\left(n_{N_{1}}-n_{N_{1}}^{\mathrm{eq}}\right)-W_{I D_{N_{1} \rightarrow \psi S}} n_{\psi} \\
-\frac{s}{H(z) z}\left[\left(n_{\psi} n_{\eta}-n_{\psi}^{\mathrm{eq}} n_{\eta}^{\mathrm{eq}}\right)\langle\sigma v\rangle_{\psi \eta \rightarrow S l}+\left(n_{\psi} n_{S}-n_{\psi}^{\mathrm{eq}} n_{S}^{\mathrm{eq}}\right)\langle\sigma v\rangle_{\psi S \rightarrow l \eta}\left(n_{\psi}-n_{\psi}^{\mathrm{eq}}\right) n_{l}^{\mathrm{eq}}\langle\sigma v\rangle_{\psi l \rightarrow \eta S}\right] \\
\frac{d n_{N_{1}}}{d z}=-D_{N_{1}}\left(n_{N_{1}}-n_{N_{1}}^{\mathrm{eq}}\right)-D_{N_{1} \rightarrow \psi S}\left(n_{N_{1}}-n_{N_{1}}^{\mathrm{eq}}\right)-\frac{s}{H(z) z}\left[\left(n_{N_{1}}^{2}-\left(n_{N_{1}}^{\mathrm{eq}}\right)^{2}\right)\langle\sigma v\rangle_{N_{1} N_{1} \rightarrow l l}+\left[n_{N_{1}} n_{\mathrm{SM}}-n_{N_{1}}^{\mathrm{eq}} n_{\mathrm{SM}}^{\mathrm{eq}}\right]\langle\sigma v\rangle_{\left.\eta l \rightarrow N_{1}\left(W^{ \pm}, Z\right)\right]}\right] \\
\frac{d n_{(B-L))_{i}}=-\epsilon_{\psi i} D_{\psi}\left(n_{\psi}-n_{\psi}^{\mathrm{eq}}\right)-\epsilon_{N_{1} i} D_{N_{1}}\left(n_{N_{1}}-n_{N_{1}}^{\mathrm{eq}}\right)-\left(W_{N_{1}} P_{1 i}+W_{\psi i} P_{\psi i}\right) n_{(B-L)_{i}}-\frac{s}{H(z) z}\left[P_{\psi i} \Gamma_{S l_{i} \rightarrow \psi \eta}+P_{\psi i} \Gamma_{l_{i} \eta \rightarrow \psi S}\right.}{\left.+P_{\psi i} \sum_{j} P_{\psi j} \Gamma_{l_{i} l_{j} \rightarrow \eta \eta}+P_{\psi i} \sum_{j} P_{\psi j} \Gamma_{l_{i} l_{j} \rightarrow N_{1} N_{1}}+P_{\psi i} \Gamma_{l_{i} \eta \rightarrow\left(N_{1} W^{ \pm}, Z\right)}+P_{\psi i} \sum_{j} \Gamma_{\eta l_{i} \rightarrow \eta^{*} \bar{l}_{j}}+P_{\psi i} \Gamma_{\psi l_{i} \rightarrow S \eta}\right] n_{(B-L)_{i}}}
\end{gathered}
$$



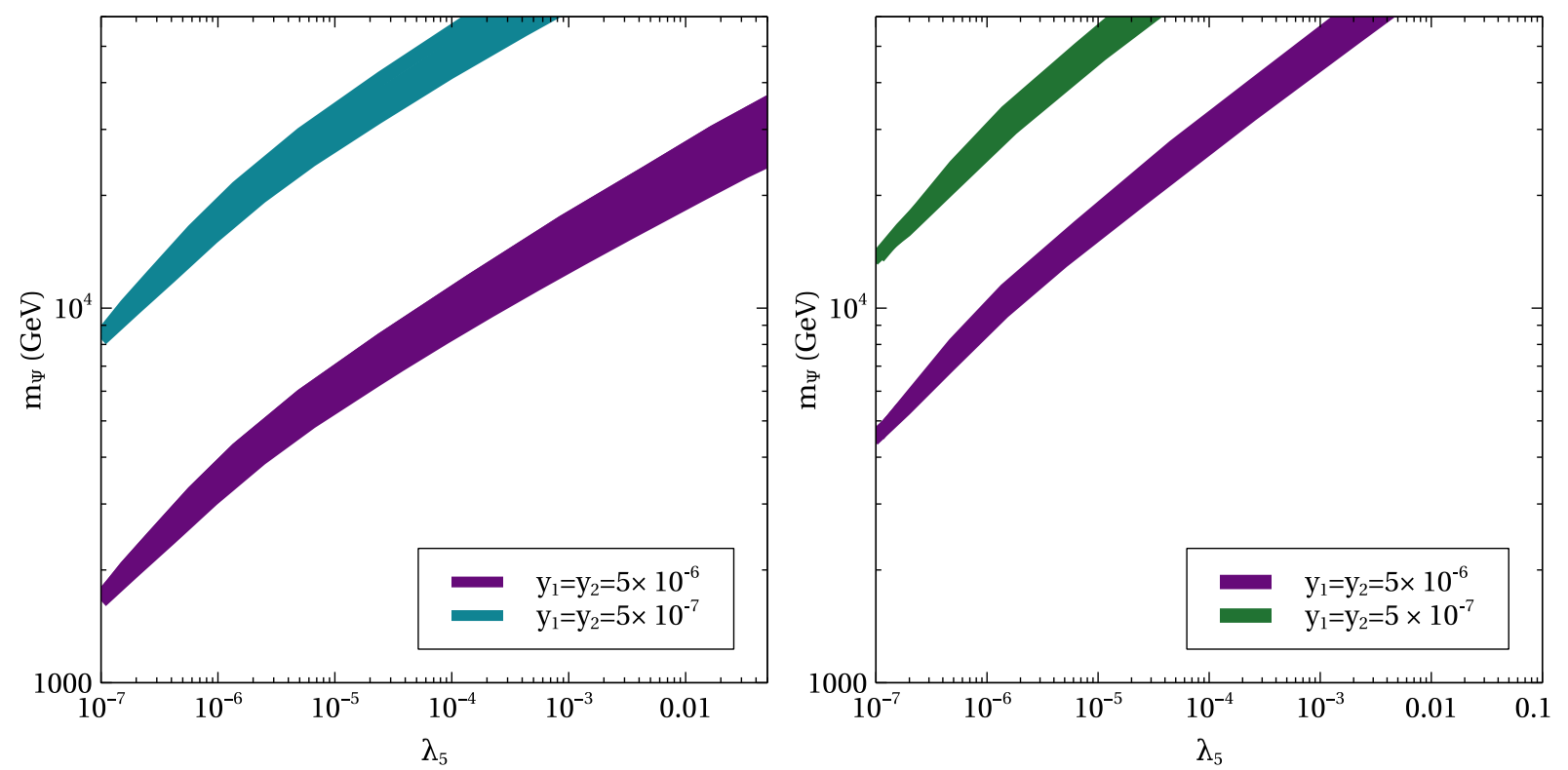

FIG. 8. The variation of $m_{\psi}$ with $\lambda_{5}$, required to satisfy the observed asymmetry using lepton flavor effects. For the left panel, $M_{1}=2 \times 10^{5} \mathrm{GeV}$ and $M_{2}=2 \times 10^{6} \mathrm{GeV}$ and for the right panel, $M_{1}=2 \times 10^{7} \mathrm{GeV}$ and $M_{2}=2 \times 10^{9} \mathrm{GeV}$. The other parameters are set at $m_{\eta}=10 \mathrm{GeV}, m_{S}=500 \mathrm{GeV}$.

where the projectors $P_{\psi i}$ and $P_{1 i}$ are defined, respectively, as

$$
\begin{gathered}
P_{\psi i}=\frac{\Gamma_{\psi \rightarrow S \eta l_{i}}}{\sum_{j} \Gamma_{\psi \rightarrow S \eta l_{j}}} . \\
P_{1 i}=\frac{\Gamma_{N_{1} \rightarrow l_{i} \eta}}{\sum_{j} \Gamma_{N_{1} \rightarrow l_{j} \eta}}
\end{gathered}
$$

In the projectors, the denominator indicates the total decay width of $\psi, N_{1}$ whereas the numerators correspond to partial decay width into a particular lepton flavor. The washout terms remain same as before. We fix the benchmarks same as the ones used in scanning the parameter space of unflavored leptogenesis. The resulting parameter space in $m_{\psi}-\lambda_{5}$ plane for both the cases are shown in Fig. 8. Comparing with scan plots for unflavored leptogenesis shown in Fig. 7, it is seen that the scale of leptogenesis can be lowered after inclusion of lepton flavor effects, as expected. Thus, successful leptogenesis can occur at a scale just above $1 \mathrm{TeV}$ for $M_{1}=$ $2 \times 10^{5} \mathrm{GeV}$ and can be as low as approximately $5 \mathrm{TeV}$ for case $M_{1}=2 \times 10^{7} \mathrm{GeV}$.

\section{Dark matter}

We briefly discuss our dark matter results in this subsection. As mentioned earlier, a two component scalar singlet and scalar doublet DM has been recently discussed in detail within a type I seesaw model [72]. Instead of showing the details in general, here we focus on possible differences due to new couplings of these two DM candidates in relation to leptogenesis and neutrino mass as discussed above. We first discuss the behavior of DM relic density with its mass for various possible combinations of relevant benchmark parameters. In Fig. 9, we show the variation in individual and total DM relic densities for different mass relations between two DM candidates. While the overall features agree with the known results of scalar singlet and scalar doublet DM, there are some interesting differences due to interconversions and coannihilations here which we highlight.

In top left panel of Fig. 9, the two DM candidates are assumed to have equal masses. The Higgs portal interactions of both the DM candidates are open due to the chosen nonzero couplings $\lambda_{6}, \lambda_{L}=\lambda_{3}+\lambda_{4}+\lambda_{5}$. Although the Higgs portal coupling of doublet DM is relatively smaller, the coannihilation channels are very efficient due to tiny mass splittings $\Delta m_{\eta_{I}}=m_{\eta_{I}}-m_{\eta_{R}}$, $\Delta m_{\eta^{ \pm}}=m_{\eta^{ \pm}}-m_{\eta_{R}}$, keeping its relic abundance suppressed compared to the singlet DM. In the top right panel plot of Fig. 9, a noticeable change in doublet DM relic abundance is observed. While all relevant couplings have the same value as those on the top left panel plot, the doublet DM relic increases as singlet DM mass is twice the mass of doublet DM and hence there can be efficient conversions from singlet to doublet DM through Higgs portal interactions. Note that in both of these plots, the direct conversion coupling $\lambda_{7}$ is switched off and hence all possible DM conversions can occur only via Higgs portal interactions. To show the effect of DM conversion more clearly, we keep the mass of doublet DM fixed in the 

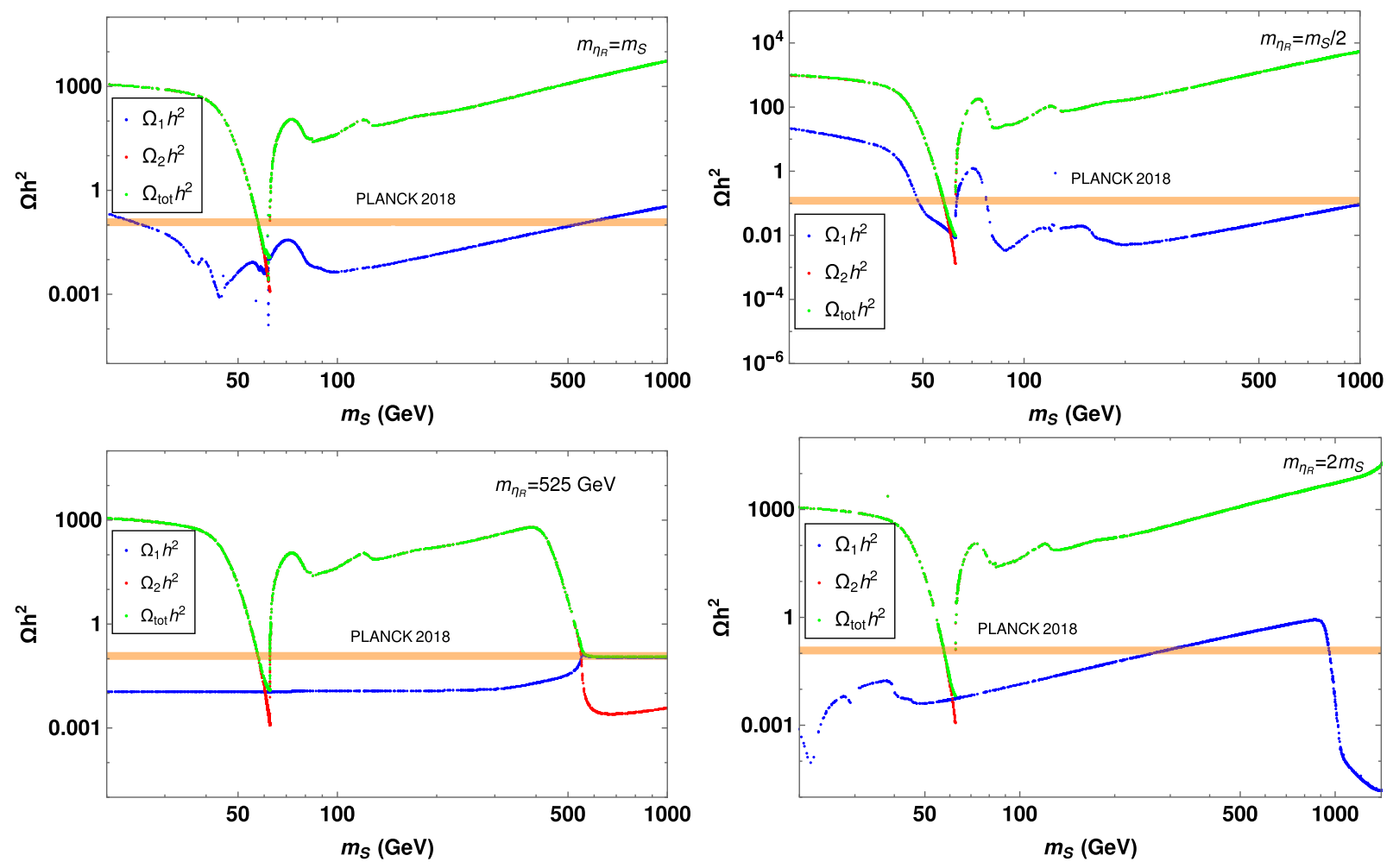

FIG. 9. Relic abundance versus DM mass for various mass relations between two DM candidates. The other parameters are fixed at the following benchmark values $\lambda_{L}=10^{-4}, \lambda_{6}=10^{-3}, \lambda_{7}=0, y_{1,2}=10^{-4}, \Delta m_{\eta_{I}}=2 \mathrm{GeV}$, and $\Delta m_{\eta_{ \pm}}=2 \mathrm{GeV}$.

bottom left panel plot of Fig. 9. As the singlet DM mass approaches the doublet DM mass, there is a sharp fall in its relic while at the same time the doublet relic increases due to relative conversions. In this plot, such conversions can occur via both Higgs portal and direct coupling $\lambda_{7}$. Finally, on the bottom right panel of Fig. 9, we show one interesting feature where doublet DM relic density suddenly drops as its mass becomes close to $1.5 \mathrm{TeV}$. This particular feature is not due to DM conversions via Higgs portal or direct coupling $\lambda_{7}$ as that can happen at any mass, given the fact that doublet mass is twice that of singlet mass all throughout. This happens due to doublet DM coannihilation with $\psi$ whose mass is fixed at $1.5 \mathrm{TeV}$. Due to this coannihilation $\eta_{R} \psi \rightarrow S \ell$, the singlet relic density also increases, though it is not as prominent as the depletion of doublet relic density in the figure.

After discussing the general features of DM relic dependence on various relevant parameters, in Fig. 10, we specifically show the effects of direct conversion coupling $\lambda_{7}$ and Yukawa coupling $y_{1,2}$ of $\psi-S-N_{1,2}$ vertices. Mass of doublet dark matter is assumed to be twice of singlet dark matter mass. Comparing top panel plots of Fig. 10 where $y_{1,2}=0$, it is seen that turning on the direct conversion coupling $\lambda_{7}$ leads to sharp fall in heavier DM relic density. Same effect is visible while comparing the bottom panel plots also where the effect of $y_{1,2} \neq 0$ is also shown leading to depletion of doublet DM relic as its mass approaches $m_{\psi}$.
To find the relevant parameter space of DM that gives rise to the observed relic density, we perform a numerical scan of the relevant parameter space favored from the requirement of successful leptogenesis. For the first case $\left(M_{1}=2 \times 10^{5} \mathrm{GeV}\right)$, the parameter space in terms of two DM masses is shown on left panel plot of Fig. 11. To be in agreement with the parameter space chosen for leptogenesis, here we fix $m_{\eta_{R}}<$ $m_{\psi}$ and vary other parameters in the range $10 \mathrm{GeV}<m_{S}$, $m_{\eta}<1000 \mathrm{GeV}, 10^{-6}<\lambda_{5}<10^{-1}, 10^{-4}<\lambda_{6}, \lambda_{7}<10^{-2}$, $10^{-7}<y_{1,2}<10^{-5}$. While singlet DM masses are evenly distributed across the range, there seems to be an upper bound on doublet DM mass near $500 \mathrm{GeV}$. This is due to the chosen mass splitting within doublet components. As earlier studies of inert scalar doublet DM shows [21,58,106-119], for such small mass splitting, the DM is overproduced in the high mass regime. While underproduction of one DM component in our model can be compensated by the second DM component, overabundance of one is difficult to reconcile with. Choosing a larger mass splitting within inert doublet components will allow more region of parameter space in terms of doublet DM mass. The right panel plot of Fig. 11 shows the spin independent DM-nucleon scattering rate of both the DM components, compared against the latest bound from Xenon1T experiment [130]. Clearly, all the points satisfy the direct detection bounds. This is due to the fact that, we have kept the Higgs portal coupling of both the DM 

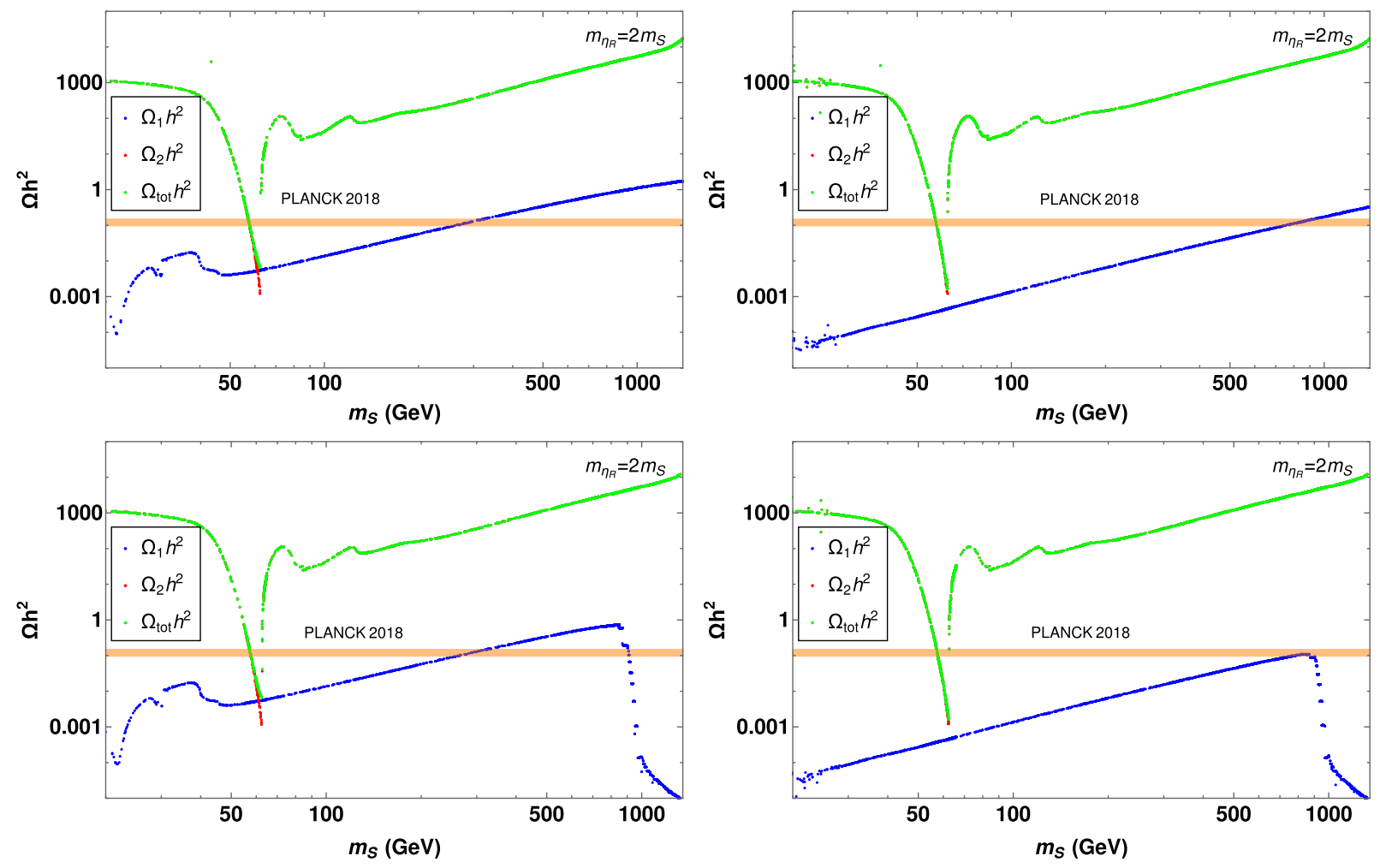

FIG. 10. Relic abundance versus DM mass showing the effects of direct conversion coupling $\lambda_{7}$ and Yukawa coupling $y_{1,2}$ of $\psi-S-N_{1,2}$ vertices. The benchmark parameters fixed for all the four plots are $\lambda_{6}=10^{-3}$ and $\lambda_{L}=10^{-4}$. The conversion coupling and the new Yukawa coupling are fixed at $y_{1,2}=0, \lambda_{7}=0$ (upper left panel plot), $y_{1,2}=0, \lambda_{7}=1$ (upper right panel plot), $y_{1,2}=10^{-2}$, $\lambda_{7}=0$ (lower left panel plot) $y_{1,2}=10^{-2}, \lambda_{7}=1$ (lower right panel plot).
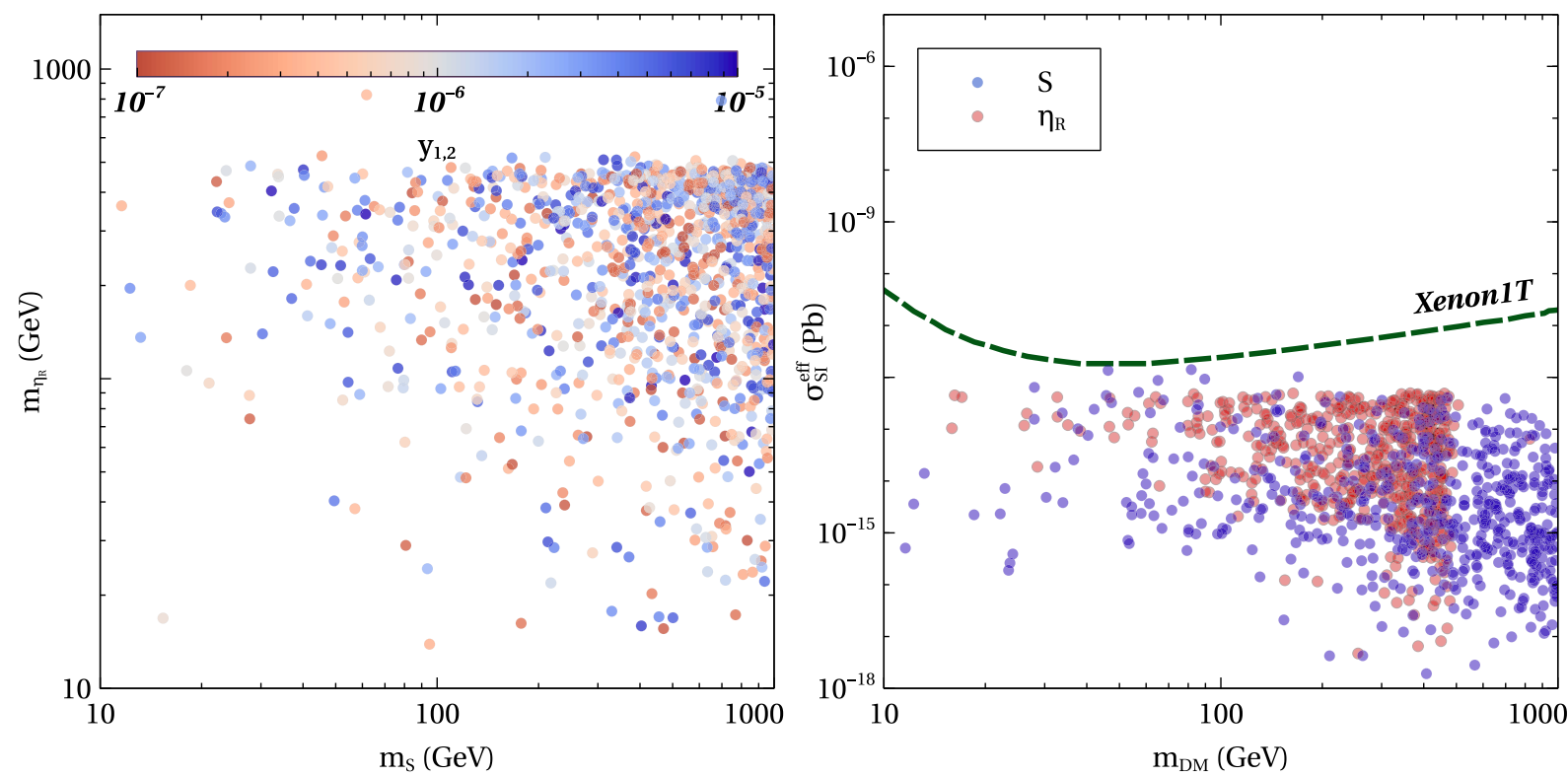

FIG. 11. Scan plot showing the parameter space in $m_{S}-m_{\eta_{R}}$ plane allowed from total DM relic abundance (left panel) and $m_{\mathrm{DM}}-\sigma_{\mathrm{SI}}$ plot for all the points satisfying the total relic (right panel). For this scan the RHN masses are set at $M_{1}=2 \times 10^{5} \mathrm{GeV}$ and $M_{2}=2 \times 10^{6} \mathrm{GeV}$. The other important parameters are randomly varied within the ranges $10^{-7}<\lambda_{5}<10^{-1}, 10^{-4}<\lambda_{6}<10^{-2}$, $10^{-4}<\lambda_{7}<10^{-2}$ and $10^{-7}<y_{1,2}<10^{-5}$. 

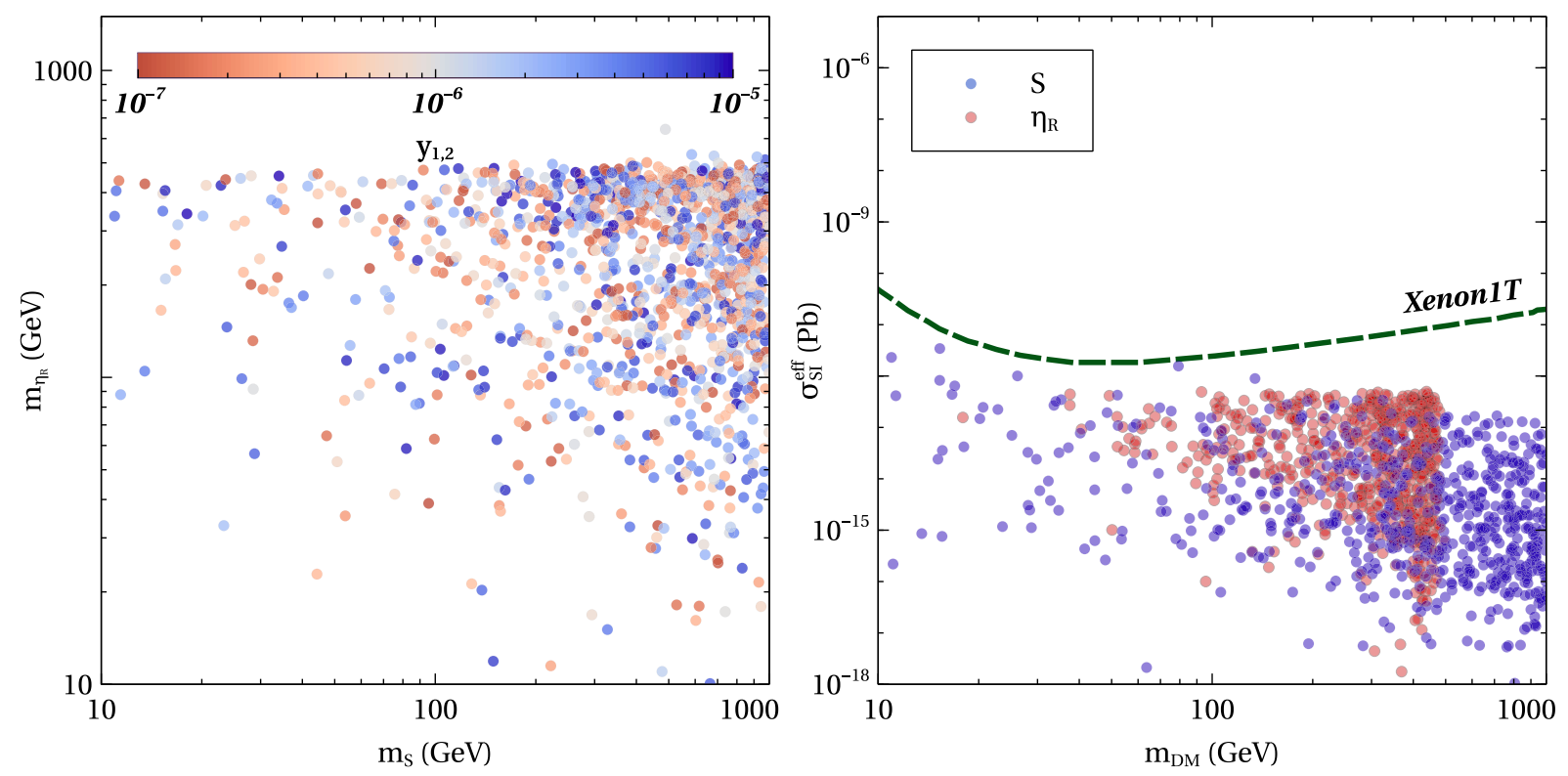

FIG. 12. Scan plot showing the parameter space in $m_{S}-m_{\eta_{R}}$ plane allowed from total DM relic abundance (left panel) and $m_{\mathrm{DM}}-\sigma_{\mathrm{SI}}$ plot for all the points satisfying the total relic (right panel). For this scan the RHN masses are set at $M_{1}=2 \times 10^{7} \mathrm{GeV}$ and $M_{2}=2 \times 10^{8} \mathrm{GeV}$. The other important parameters are randomly varied within the ranges $10^{-7}<\lambda_{5}<10^{-1}, 10^{-4}<\lambda_{6}<10^{-2}$, $10^{-4}<\lambda_{7}<10^{-2}$ and $10^{-7}<y_{1,2}<10^{-5}$.

candidates fixed at small value. We varied $10^{-6}<\lambda_{6}<$ $10^{-2}$ and fixed $\lambda_{L}=10^{-3}$. Since tree level DM-nucleon scattering arises through Higgs portal couplings only, the corresponding rates remain low enough to survive Xenon1T bounds. The color code on left panel plot of Fig. 11 shows the value of $y=\left|y_{1}\right|=\left|y_{2}^{*}\right|$. Similar scan plot for case 2 is shown on the left panel of Fig. 12. While we notice a similar upper bound on doublet DM mass due to chosen mass splitting, the parameter space remains safe from direct detection bounds.

\section{CONCLUSION}

We have proposed a model to implement the idea of leptogenesis from three body decay of a heavy particle where nonzero $C P$ asymmetry arises due to interference of multiple three body decay diagrams with resummed propagators along with dark matter. Adopting a minimal framework to implement the idea, we augment the standard model of particle physics by three singlet fermions and two scalar fields: one singlet and one doublet. While two of these singlet fermions and the additional scalar doublet help in generating light neutrino masses one loop level, the other two particles help in realizing the desired three body decay leptogenesis. The two singlet fermions taking part in radiative neutrino mass generation also act like mediators in two different three body decay diagrams the interference of which results in the required nonzero $C P$ asymmetry. It turns out that this setup automatically gives rise to a two component dark matter scenario in terms of scalar singlet and neutral component of scalar doublet. After deriving the particle spectrum of the model and applying the theoretical as well as experimental bounds, we calculate the $C P$ asymmetry from three body decay of heavy singlet fermion by considering interference of two different diagrams. We then solve the Boltzmann equations relevant for leptogenesis incorporating the sources of lepton asymmetry as well as washouts to obtain the parameter space that can give rise to successful leptogenesis. While both two body decay of right handed neutrino $N_{1}$ (similar to the scotogenic model) and three body decay of the new singlet fermion $\psi$ introduced in our model can contribute to lepton asymmetry, we check that in the low scale leptogenesis scenario we focus, the contribution from two body decay remains subdominant. After analyzing the role or effects of some key parameters on generation of lepton asymmetry, we performed a numerical scan and show that successful leptogenesis can occur at a scale as low as $3 \mathrm{TeV}$. This is a factor of around 3 times lower than the scale of leptogenesis in the minimal scotogenic model considering two body decay of hierarchical heavy neutrinos studied in earlier works [21-30]. In fact, we also checked that after incorporating lepton flavor effects, the scale can be as low as $2 \mathrm{TeV}$. Such low scale leptogenesis possibility could have tantalizing prospects of being probed at ongoing or near future experiments. This difference in scale of leptogenesis from minimal scotogenic model arises due to three body decay as a dominant source and also due to the freedom in choosing $y_{\alpha} \psi N_{\alpha} S$ coupling in (2) which does not play any role in generating light neutrino masses. Lowering of leptogenesis scale due to introduction of such new couplings (not related to origin of neutrino mass) have 
been explored in earlier works also. For example, in [141], the scale of leptogenesis in a scalar singlet extension of type I seesaw model was shown to be as low as $500 \mathrm{GeV}$ even with hierarchical right handed neutrinos which is significantly lower than the scale of leptogenesis in usual type I seesaw model.

After finding the parameter space that gives rise to successful $\mathrm{TeV}$ scale leptogenesis, we calculate the relic abundance of two DM components. Since such two component scalar DM have been already studied in earlier works, we focus primarily on the role of new parameters involving the two DM candidates in our model which also play nontrivial roles in leptogenesis. We first analyze these effects with benchmark choices of parameters and finally show the parameter space of two DM masses that is consistent with correct relic abundance and direct detection rates. Such a low scale model with two component DM, successful leptogenesis and light neutrino masses should face further scrutiny with future data from collider, neutrino, cosmology as well as rare decay experiments looking for charged lepton flavor violation, neutrinoless double beta decay etc. While neutrinoless double beta decay contribution will effectively arise from light neutrino contributions only and will remain below the current experimental sensitivity of KamLAND-Zen experiment, i.e., $\left|m_{e e}\right| \leq$ (0.061-0.165) eV [142] for vanishing lightest neutrino mass. While charged lepton flavor violation like $\mu \rightarrow e \gamma$, $\mu \rightarrow 3 e$ and $\mu \rightarrow e$ (Ti) conversion in scotogenic models can be sizeable and saturate experimental upper bounds on corresponding branching ratios for fermion DM scenario $[65,143,144]$, in our model they are likely to be suppressed as the singlet fermions $N_{1,2}$ are heavier than the scale of leptogenesis. Another interesting prospect of probing our model can be in the form of gravitational waves from a strongly first order phase transition (SFOPT). In a recent work [144], it was shown that in the minimal scotogenic model, the criteria of SFOPT constrains the scalar sector a lot, leading to a scalar DM parameter space in tension with direct detection bounds. Due to the presence of an additional singlet scalar in our model whose mass is not as constrained as the inert doublet components, the SFOPT criteria is likely to be satisfied with more freedom. We leave a detailed study of this model from SFOPT point of view to future works.

\section{ACKNOWLEDGMENTS}

D. B. acknowledges the support from Early Career Research Award from DST-SERB, Government of India (reference number: ECR/2017/001873).

\section{APPENDIX A: $C P$ ASYMMETRY FROM THREE BODY DECAY OF $\psi$}

Let us start deriving the most general expression of asymmetry from an out of equilibrium process. The usual amplitude for a process is given as
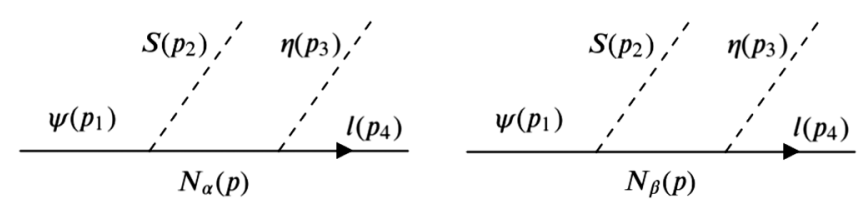

FIG. 13. Two processes for the three body decay.

$$
i \mathcal{M}_{i \rightarrow f}=\mathcal{E} \times \mathcal{A} \times \omega,
$$

where $\mathcal{E}$ comprises of all the couplings and $\omega$ comprises of all the wave functions of outgoing and incoming particles. Finally $\mathcal{A}$ contains all the rest of the term of the amplitude. For a nonzero $C P$ asymmetry to be created one need at least two amplitudes for a process as shown in Fig. 13.

Then the total amplitude for the process can be written as,

$$
i \mathcal{M}_{i \rightarrow f}=\left[\mathcal{E}_{1} \mathcal{A}_{1}+\mathcal{E}_{2} \mathcal{A}_{2}\right] \omega .
$$

Similarly, the amplitude for the process corresponding to its antiparticle counterpart is given as,

$$
i \mathcal{M}_{\bar{i} \rightarrow \bar{f}}=\left[\mathcal{E}_{1}^{*} \mathcal{A}_{1}+\mathcal{E}_{2}^{*} \mathcal{A}_{2}\right] \omega^{\dagger} .
$$

The corresponding amplitude squared terms are given as,

$$
\begin{aligned}
\left|\mathcal{M}_{i \rightarrow f}\right|^{2}= & \left(\left|\mathcal{E}_{1}\right|^{2}\left|\mathcal{A}_{1}\right|^{2}+\left|\mathcal{E}_{2}\right|^{2}\left|\mathcal{A}_{2}\right|^{2}+2 \operatorname{Re}\left[\mathcal{E}_{1}^{*} \mathcal{E}_{2}\right] \operatorname{Re}\left[\mathcal{A}_{1}^{*} \mathcal{A}_{2}\right]\right. \\
& \left.+2 \operatorname{Im}\left[\mathcal{E}_{1}^{*} \mathcal{E}_{2}\right] \operatorname{Im}\left[\mathcal{A}_{1}^{*} \mathcal{A}_{2}\right]\right)|\omega|^{2} \\
\left|\mathcal{M}_{\bar{i} \rightarrow \bar{f}}\right|^{2}= & \left(\left|\mathcal{E}_{1}\right|^{2}\left|\mathcal{A}_{1}\right|^{2}+\left|\mathcal{E}_{2}\right|^{2}\left|\mathcal{A}_{2}\right|^{2}+2 \operatorname{Re}\left[\mathcal{E}_{1} \mathcal{E}_{2}^{*}\right] \operatorname{Re}\left[\mathcal{A}_{1}^{*} \mathcal{A}_{2}\right]\right. \\
& \left.+2 \operatorname{Im}\left[\mathcal{E}_{1} \mathcal{E}_{2}^{*}\right] \operatorname{Im}\left[\mathcal{A}_{1}^{*} \mathcal{A}_{2}\right]\right)|\omega|^{2}
\end{aligned}
$$

and therefore, the asymmetry is calculated to be,

$\delta=\left|\mathcal{M}_{i \rightarrow f}\right|^{2}-\left|\mathcal{M}_{\bar{i} \rightarrow \bar{f}}\right|^{2}=-4 \operatorname{Im}\left[\mathcal{E}_{1}^{*} \mathcal{E}_{2}\right] \operatorname{Im}\left[\mathcal{A}_{1}^{*} \mathcal{A}_{2}\right]|\omega|^{2}$.

In the following subsections we first calculate the $C P$ asymmetry by using the resummed propagators and then verify the same by tree-loop diagram interference calculation. We have adopted the two spinor notations from [145] throughout the derivations.

\section{Calculation of the $C P$ asymmetry using resummed propagator}

Before doing the $C P$ asymmetry calculation using resummed propagator let us calculate the resummed propagators first. In Fig. 14 we show the diagrammatic representations of the full loop corrected propagators for two component fermions. For a detailed calculation on resummed propagator please see [145].

The full propagators can be organized in terms of one particle irreducible (1PI) self-energy functions [145]. 

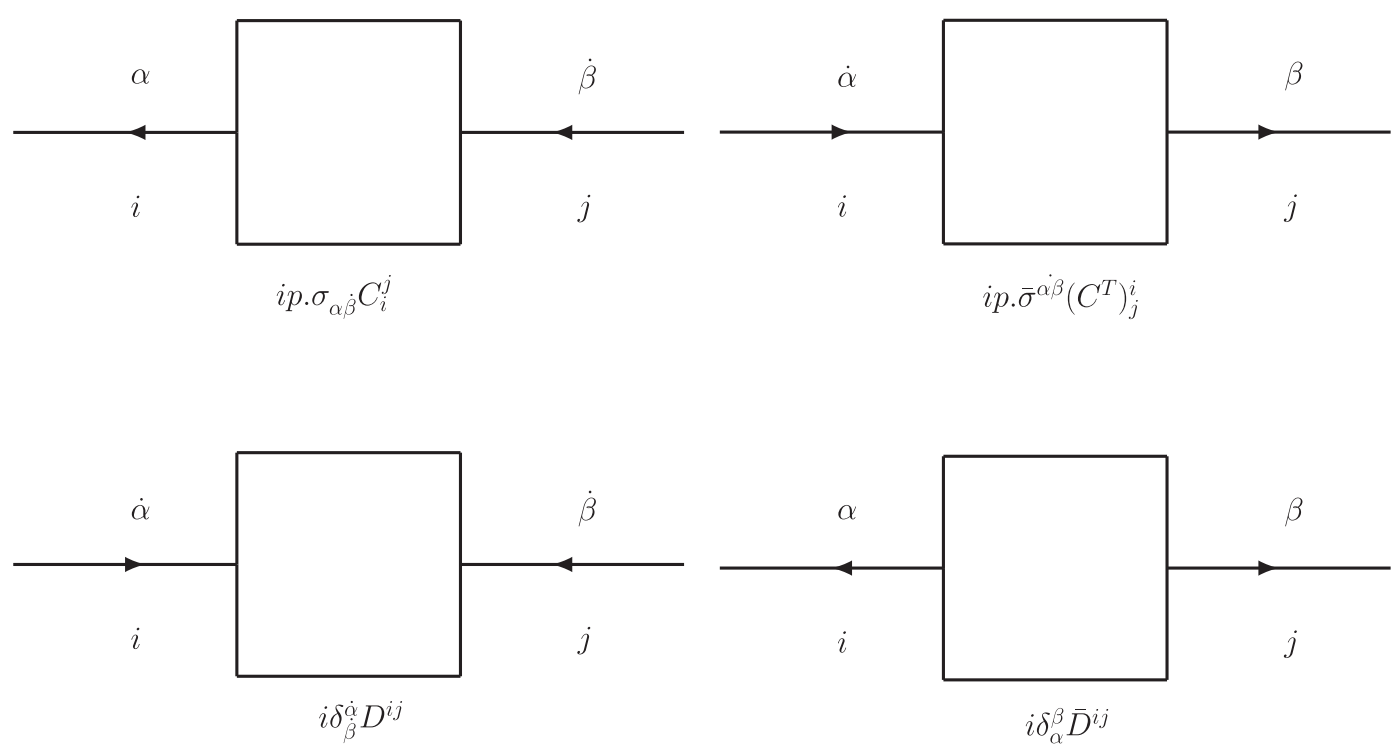

FIG. 14. The full loop corrected propagators for two component fermions are associated with functions $C\left(p^{2}\right)_{i}^{j}$ and its matrix transpose, $D\left(p^{2}\right)^{i j}$ and $\bar{D}\left(p^{2}\right)_{i j}$. The square boxes represent all the sum of all connected Feynman diagrams, with external legs included. The four-momentum $p$ flows from right to left.

These are defined as the sum of Feynman diagrams to all orders in perturbation theory (with the corresponding tree level graph excluded) that contribute to the 1PI two-point Green functions.

$\left(\begin{array}{cc}i \overline{\mathbf{D}} & i p . \sigma \mathbf{C} \\ i p . \bar{\sigma} \mathbf{C}^{T} & i \mathbf{D}\end{array}\right)=\left(\begin{array}{cc}i(m+\Omega) & -i p . \sigma\left(1-\Xi^{T}\right) \\ -i p . \bar{\sigma}(1-\Xi) & i(\bar{m}+\bar{\Omega})\end{array}\right)^{-1}$

The right-hand side of the above equation can be evaluated by employing the following identity for the inverse of a block diagonal matrix,

$\left(\begin{array}{ll}P & Q \\ R & S\end{array}\right)^{-1}=\left(\begin{array}{cc}\left(P-Q S^{-1} R\right)^{-1} & \left(R-S Q^{-1} P\right)^{-1} \\ \left(Q-P R^{-1} S\right)^{-1} & \left(S-R P^{-1} Q\right)^{-1}\end{array}\right)$

under the assumption that all inverses, appearing in the Eq. (A8) exist. Applying this result to the Eq. (A7) we get,

$$
\begin{gathered}
C^{-1}=s(1-\Xi)-(\bar{m}+\bar{\Omega})\left(1-\Xi^{T}\right)^{-1}(m+\Omega), \\
D^{-1}=s(1-\Xi)(m+\Omega)^{-1}\left(1-\Xi^{T}\right)-(\bar{m}+\bar{\Omega}), \\
\bar{D}^{-1}=s\left(1-\Xi^{T}\right)(\bar{m}+\bar{\Omega})^{-1}(1-\Xi)-(m+\Omega),
\end{gathered}
$$

where $s=p^{2}$. Taking the inverse and keeping the calculation up to one-loop order,

$$
\begin{aligned}
C & =\left[s(1-\Xi)\left(1-\Xi^{T}\right)-(\bar{m} m+\bar{m} \Omega+m \bar{\Omega})\right]^{-1}\left(1-\Xi^{T}\right)=C^{T} \\
& =\frac{1-\Xi^{T}}{s\left(1-\left(\Xi+\Xi^{T}\right)\right)-(\bar{m} m+\bar{m} \Omega+m \bar{\Omega})} \\
& =\frac{1-\Xi^{T}}{\left[s-m^{2}-\left(s\left(\Xi+\Xi^{T}\right)+m \bar{\Xi}+\bar{m} \Omega\right)\right]} \\
& =\frac{1-\Xi^{T}}{\left(s-m^{2}\right)\left[1-\frac{\left(s\left(\Xi+\Xi^{T}\right)+m \bar{\Omega}+\bar{m} \Omega\right)}{s-m^{2}}\right]} \\
& =\frac{1-\Xi^{T}}{\left(s-m^{2}\right)}\left[1+\frac{s\left(\Xi+\Xi^{T}\right)+m \bar{\Omega}+\bar{m} \Omega}{s-m^{2}}\right] \\
& =\frac{1}{s-m^{2}}+\frac{s \Xi+m^{2} \Xi^{T}+m \bar{\Omega}+\bar{m} \Omega}{\left(s-m^{2}\right)^{2}}
\end{aligned}
$$

Similarly, for the mass insertion section it can found out that,

$$
D=\frac{m}{s-m^{2}}+\frac{s\left(m \Xi+m \Xi^{T}+\Omega\right)+m^{2} \bar{\Omega}}{\left(s-m^{2}\right)^{2}}
$$

$\bar{D}=\frac{\bar{m}}{s-m^{2}}+\frac{\bar{m} s\left(\Xi+\Xi^{T}\right)+s \bar{\Omega}+\bar{m}^{2} \Omega}{\left(s-m^{2}\right)^{2}}=(D)^{*}$.

To calculate the $C P$ asymmetry let us consider the tree level diagrams with the resummed propagators. The amplitudes for the tree level diagrams with the resummed propagators (denoted by subscript $i$ and $j$ ) can be written as

$$
\mathcal{M}_{i}=D_{i i} x_{l}^{\dagger} y_{\Psi}^{\dagger} y_{i}^{*} h_{i \alpha}^{*}+C_{i i} x_{l}^{\dagger} \bar{\sigma} p x_{\Psi} y_{i} h_{i \alpha}^{*},
$$




$$
\mathcal{M}_{j}=D_{j j} x_{l}^{\dagger} y_{\Psi}^{\dagger} y_{j}^{*} h_{j \alpha}^{*}+C_{j j} x_{l}^{\dagger} \bar{\sigma} p x_{\Psi} y_{j} h_{j \alpha}^{*} .
$$

With the total amplitude $\mathcal{M}$ being,

$$
\mathcal{M}=\mathcal{M}_{i}+\mathcal{M}_{j}
$$

Taking the interference, the asymmetry in the amplitude level can be found to be

$$
\begin{aligned}
\delta= & |\mathcal{M}|^{2}-|\overline{\mathcal{M}}|^{2} \\
= & \operatorname{Im}\left[y_{i}^{*} h_{i \alpha}^{*} y_{j} h_{j \alpha}\right] \operatorname{Im}\left[D_{i} D_{j}^{*}\right] \operatorname{Tr}\left[p_{l} \cdot \sigma p_{\Psi} \cdot \bar{\sigma}\right] \\
& +\operatorname{Im}\left[y_{i} y_{j}^{*} h_{i \alpha}^{*} h_{j \alpha}\right] \operatorname{Im}\left[C_{i} C_{j}^{*}\right] \operatorname{Tr}\left[p_{l} \cdot \sigma \bar{\sigma} \cdot p p_{\Psi} \cdot \sigma \bar{\sigma} \cdot p\right] \\
& +\left[\operatorname{Im}\left[y_{i} h_{i \alpha}^{*} y_{j} h_{j \alpha}\right] \operatorname{Im}\left[C_{i} D_{j}^{*}\right]+\operatorname{Im}\left[y_{i}^{*} h_{i \alpha}^{*} y_{j}^{*} h_{j \alpha}\right]\right. \\
& \left.\times \operatorname{Im}\left[D_{i} C_{j}^{*}\right]\right] \operatorname{Tr}\left[p_{l} \cdot \sigma \bar{\sigma} \cdot p\right] m_{\Psi} .
\end{aligned}
$$

The relevant quantities coming from the interference of the resummed propagators can be found out to be (keeping the calculation up to one-loop order),

$$
\begin{aligned}
& \operatorname{Im}\left[D_{i} D_{j}^{*}\right] \\
& =\operatorname{Im}\left[\frac{M_{i}}{p^{2}-M_{i}^{2}}\left(\frac{p^{2}\left(M_{j} \Xi_{j}^{*}+M_{j} \Xi_{j}\right)+p^{2} \Omega_{j}^{*}+M_{j} \bar{\Omega}_{j}^{*}}{\left(p^{2}-M_{j}^{2}\right)^{2}}\right)\right] \\
& \quad+\operatorname{Im}\left[\frac{M_{j}}{p^{2}-M_{j}^{2}}\left(\frac{p^{2}\left(M_{i} \Xi_{i}+M_{i} \Xi_{i}^{*}\right)+p^{2} \Omega_{j}+M_{i}^{2} \Omega_{i}^{*}}{\left(p^{2}-M_{i}^{2}\right)^{2}}\right)\right]
\end{aligned}
$$

Similarly,

$$
\begin{aligned}
\operatorname{Im}\left[C_{i} C_{j}^{*}\right]= & \operatorname{Im}\left[\frac{p^{2} \Xi_{j}^{*}+M_{j}^{2} \Xi_{j}+M_{j} \Omega_{j}+M_{j} \Omega_{j}^{*}}{\left(p^{2}-M_{i}^{2}\right)\left(p^{2}-M_{j}^{2}\right)^{2}}\right. \\
& \left.+\frac{p^{2} \Xi_{i}+M_{i} \Xi_{i}^{*}+M_{i} \Omega_{i}^{*}+M_{i} \Omega_{i}}{\left(p^{2}-M_{i}^{2}\right)^{2}\left(p^{2}-M_{j}^{2}\right)^{2}}\right],
\end{aligned}
$$

$$
\begin{aligned}
\operatorname{Im}\left[C_{i} D_{j}^{*}\right]= & \operatorname{Im}\left[\frac{p^{2}\left(M_{i} \Xi_{i}^{*}+M_{i} \Xi_{i}^{*}+\Omega_{i}^{*}\right)+M_{i} \Omega_{i}^{*}}{\left(p^{2}-M_{j}^{2}\right)\left(p^{2}-M_{i}^{2}\right)^{2}}\right. \\
& \left.+\frac{M_{i}\left(p^{2} \Xi_{j}^{*}+M_{j}^{2} \Xi_{j}+M_{j} \Omega_{j}+M_{j} \Omega_{j}^{*}\right)}{\left(p^{2}-M_{i}^{2}\right)\left(p^{2}-M_{j}^{2}\right)^{2}}\right],
\end{aligned}
$$

$$
\begin{aligned}
\operatorname{Im}\left[D_{i} C_{j}^{*}\right]= & \operatorname{Im}\left[\frac{p^{2}\left(M_{i} \Xi_{i}+M_{i} \Xi_{i}^{*}+\Omega_{i}\right)+M_{i}^{2} \Omega_{j}^{*}}{\left(p^{2}-M_{j}^{2}\right)\left(p^{2}-M_{i}^{2}\right)^{2}}\right. \\
& \left.+\frac{M_{i}\left(p^{2} \Xi_{j}^{*}+M_{j}^{2} \Xi_{j}+M_{j} \Omega_{j}+M_{j} \Omega_{j}^{*}\right)}{\left(p^{2}-M_{i}^{2}\right)\left(p^{2}-M_{j}^{2}\right)^{2}}\right] .
\end{aligned}
$$

For our case the self-energy functions in two component spinor notation are diagrammatically shown in Figs. 15-17 and they can be found out to be,
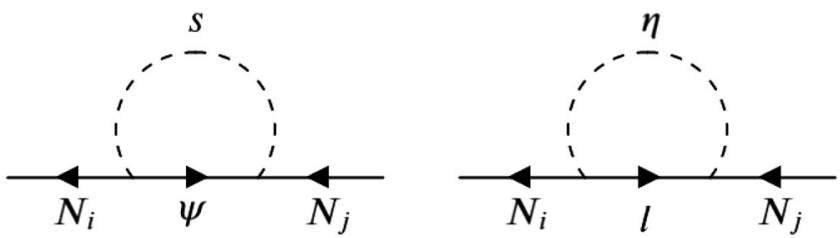

FIG. 15. Diagrammatic representation of $(\Xi)_{i}^{j}$.
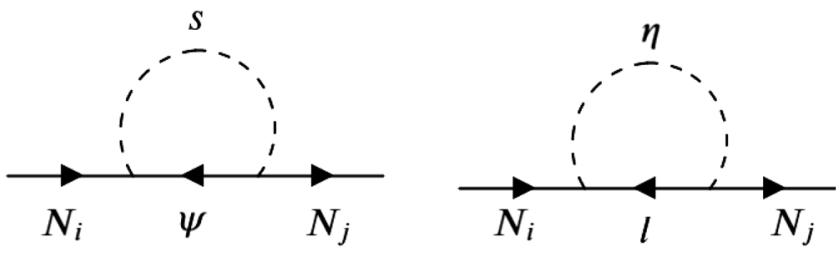

FIG. 16. Diagrammatic representation of $(\Xi)_{j}^{i}$.
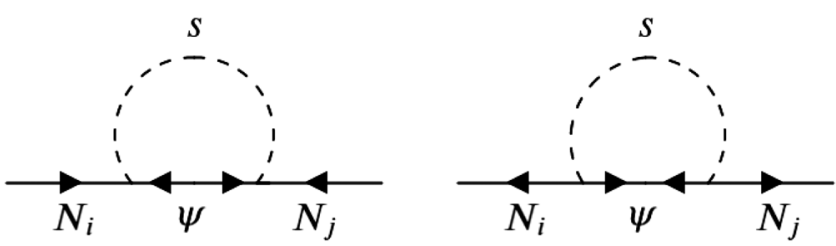

FIG. 17. Diagrammatic representation of $(\Omega)^{i j}$ and $(\bar{\Omega})_{i j}$.

$$
\begin{aligned}
(\Xi)_{j}^{i}= & \frac{y_{i}^{*} y_{j}}{16 \pi^{2}} I_{\mathrm{FS}}\left(s, m_{\Psi}^{2}, m_{S}^{2}\right) \\
& +\sum_{l} \frac{h_{i l}^{*} h_{j l}}{16 \pi^{2}} I_{\mathrm{FS}}\left(s, m_{l}^{2}, m_{\eta}^{2}\right)=(\Xi), \\
\left(\Xi^{T}\right)_{j}^{i}= & \frac{y_{i} y_{j}^{*}}{16 \pi^{2}} I_{\mathrm{FS}}\left(s, m_{\Psi}^{2}, m_{S}^{2}\right) \\
& +\sum_{l} \frac{h_{i l} h_{j l}^{*}}{16 \pi^{2}} I_{\mathrm{FS}}\left(s, m_{l}^{2}, m_{\eta}^{2}\right)=\left(\Xi^{T}\right), \\
(\Omega)^{i j} & =\frac{y_{i} y_{j}}{16 \pi^{2}} m_{\Psi} I_{\overline{\mathrm{FS}}}\left(s, m_{\Psi}^{2}, m_{S}^{2}\right)=(\Omega), \\
(\bar{\Omega})_{i j} & =\frac{y_{i}^{*} y_{j}^{*}}{16 \pi^{2}} m_{\Psi} I_{\overline{\mathrm{FS}}}\left(s, m_{\Psi}^{2}, m_{S}^{2}\right)=(\bar{\Omega}) .
\end{aligned}
$$

Here,

$$
\begin{gathered}
I_{\mathrm{FS}}(s, x, y)=\frac{1}{2 \epsilon}+\left[\frac{(s+x-y) B_{0}(s ; x, y)+A_{0}(x)-A_{0}(y)}{2 s}\right] . \\
I_{\overline{\mathrm{FS}}}(s, x, y)=\frac{1}{\epsilon}-B_{0}(s ; x, y)
\end{gathered}
$$

where, $A_{0}$ and $B_{0}$ are the Passarino-Veltman functions. For identical right handed neutrinos $N_{i}$ on external legs, we use 

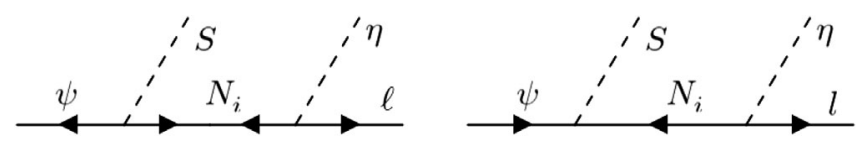

FIG. 18. Tree level diagram contributing to the three body decay of $\psi$.

the notation $\Xi_{i}, \Omega_{i}$, as seen in Eq. (A19), (A20), (A21), (A22) mentioned above.

Using Eqs. (A19), (A20), (A21), and (A22) in Eq. (A18) we get final expression of the $C P$ asymmetry. To find the $C P$ asymmetry parameter $\epsilon_{\psi}$, defined in Eq. (17) we perform the three body phase space integration numerically without any assumption. While we do not write the final $C P$ asymmetry expression in this subsection, it is identical to the one derived using the interference of tree-loop diagrams as we show in the next subsection.

\section{2. $C P$ asymmetry calculation from tree-loop interference}

In this Appendix we calculate asymmetry parameter from the interference of tree and one-loop diagrams. The relevant diagrams in the tree level are shown in Fig. 18. The amplitudes for the tree level diagram can be written as,

$i \mathcal{M}_{0}^{i}=y_{i}^{*} h_{i \alpha}^{*} \frac{x_{l}^{\dagger} y_{\Psi}^{\dagger}}{\left(p^{2}-M_{i}^{2}\right)} M_{i}+y_{i} h_{i \alpha}^{*} \frac{x_{l}^{\dagger} \bar{\sigma} \cdot p x_{\Psi}}{\left(p^{2}-M_{i}^{2}\right)}$.

For a Majorana fermion $\psi$ there are two sets of diagrams contributing to the three body decay at one-loop. These diagrams are shown in Figs. 19 and 20 respectively. For the diagrams in Fig. 19, the corresponding amplitudes can be written as,

$$
\begin{aligned}
i \mathcal{M}_{j}^{1^{\prime}}= & y_{j}^{*} h_{j \alpha}^{*} \frac{x_{l}^{\dagger} \bar{\sigma} \cdot p \sigma \cdot p y_{\Psi}^{\dagger}(\Xi)_{j}^{T} M_{j}}{\left(p^{2}-M_{j}^{2}\right)^{2}}+y_{j}^{*} h_{j \alpha}^{*} \frac{x_{l}^{\dagger} \bar{\sigma} \cdot p(\Omega)_{j} \sigma \cdot p y_{\Psi}^{\dagger}}{\left(p^{2}-M_{j}^{2}\right)^{2}} \\
& +y_{j}^{*} h_{j \alpha}^{*} \frac{x_{l}^{\dagger} \bar{\sigma} \cdot p \sigma \cdot p y_{\Psi}^{\dagger} M_{j}(\Xi)_{j}}{\left(p^{2}-M_{j}^{2}\right)^{2}}+y_{j}^{*} h_{j \alpha}^{*} \frac{x_{l}^{\dagger} M_{j}^{2}(\bar{\Omega})_{j} y_{\Psi}^{\dagger}}{\left(p^{2}-M_{j}^{2}\right)^{2}} \\
= & y_{j}^{*} h_{j \alpha}^{*} \frac{x_{l}^{\dagger} y_{\Psi}^{\dagger}}{\left(p^{2}-M_{j}^{2}\right)}\left[p ^ { 2 } \left[M_{j}(\Xi)_{j}^{T}+M_{j}(\Xi)_{j}\right.\right. \\
& \left.\left.+(\Omega)_{j}\right]+M_{j}^{2}(\bar{\Omega})_{j}\right] .
\end{aligned}
$$
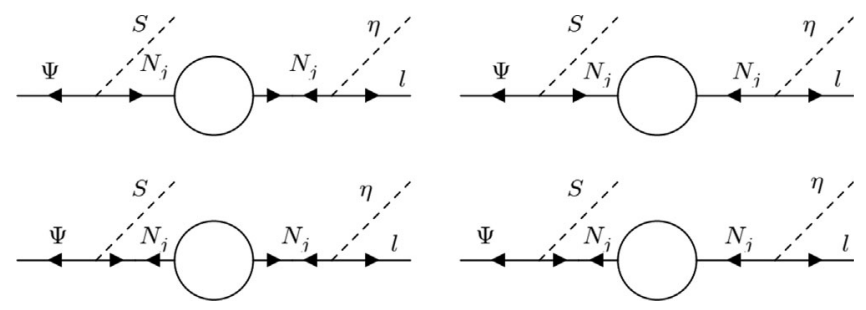

FIG. 19. Feynman diagrams contributing to the three body decay of $\Psi$ at one-loop level.
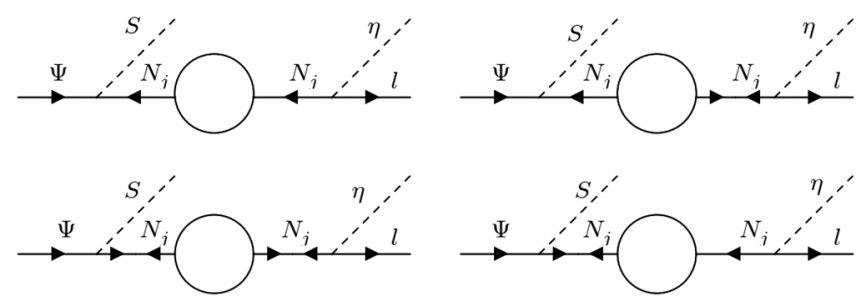

FIG. 20. Feynman diagrams contributing to the three body decay of $\Psi$ at one-loop level.

Similarly, for the other set of four one-loop diagrams in Fig. 20, the amplitude can be written as,

$$
\begin{aligned}
i \mathcal{M}_{j}^{1^{\prime \prime}}= & y_{j} h_{j \alpha}^{*} \frac{x_{l}^{\dagger} \sigma^{-} p \sigma \cdot p \bar{\sigma} \cdot p(\Xi)_{j} x_{\Psi}}{\left(p^{2}-M_{j}^{2}\right)^{2}}+y_{j} h_{j \alpha}^{*} \frac{x_{l}^{\dagger} \bar{\sigma} \cdot p M_{j}(\bar{\Omega})_{j} x_{\Psi}}{\left(p^{2}-M_{j}^{2}\right)^{2}} \\
& +y_{j} h_{j \alpha}^{*} \frac{x_{l}^{\dagger} \bar{\sigma} \cdot p x_{\Psi} M_{j}^{2}(\Xi)_{j}^{T}}{\left(p^{2}-M_{j}^{2}\right)^{2}}+y_{j} h_{j \alpha}^{*} \frac{x_{l}^{\dagger} \bar{\sigma} \cdot p x_{\Psi} M_{j}(\Omega)_{j}}{\left(p^{2}-M_{j}^{2}\right)^{2}} \\
= & y_{j} h_{j \alpha}^{*} \frac{x_{l}^{\dagger} \bar{\sigma} \cdot p x_{\Psi}}{\left(p^{2}-M_{j}^{2}\right)}\left[p^{2}(\Xi)_{j}+M_{j}^{2}(\Xi)_{j}^{T}+M_{j}(\Omega)_{j}\right. \\
& \left.+M_{j}(\bar{\Omega})_{j}\right] .
\end{aligned}
$$

Therefore, the total amplitude for the decay at one-loop level can be written as,

$$
\mathcal{M}_{j}^{1}=\mathcal{M}_{j}^{1^{\prime}}+\mathcal{M}_{j}^{1^{\prime \prime}}
$$

The asymmetry parameter is given by 


$$
\begin{aligned}
& \delta=|\mathcal{M}|^{2}-|\overline{\mathcal{M}}|^{2}=4 \operatorname{Im}\left[\mathcal{M}_{i}^{0} \mathcal{M}_{j}^{1 *}+\mathcal{M}_{i}^{1} \mathcal{M}_{j}^{0 *}\right] \\
& =4 \operatorname{Im}\left[y_{i}^{*} h_{i \alpha}^{*} y_{j} h_{j \alpha}\right]\left[\frac{\operatorname{Im}\left[M_{i}\left(p^{2}\left[M_{j}(\Xi)_{j}^{T}+M_{j}(\Xi)_{j}^{*}+\Omega_{j}^{*}\right]+M_{j}^{2} \bar{\Omega}_{j}^{*}\right)\right]}{\left(p^{2}-M_{i}^{2}\right)\left(p^{2}-M_{j}^{2}\right)^{2}}\right] \operatorname{Tr}\left[p_{l} \cdot \sigma p_{\Psi} . \bar{\sigma}\right] \\
& +4 \operatorname{Im}\left[y_{i}^{*} h_{i \alpha}^{*} y_{j} h_{j \alpha}\right]\left[\frac{\operatorname{Im}\left[M_{j}\left(p^{2}\left[M_{i}(\Xi)_{i}^{T}+M_{i}(\Xi)_{i}+\Omega_{i}\right]+M_{i}^{2} \bar{\Omega}_{i}\right)\right]}{\left(p^{2}-M_{j}^{2}\right)\left(p^{2}-M_{i}^{2}\right)^{2}}\right] \operatorname{Tr}\left[p_{l} \cdot \sigma p_{\Psi} \cdot \bar{\sigma}\right] \\
& +4 \operatorname{Im}\left[y_{i} h_{i \alpha}^{*} y_{j}^{*} h_{j \alpha}\right]\left[\frac{\operatorname{Im}\left[p^{2}(\Xi)_{j}^{*}+M_{j}^{2}(\Xi)_{j}+M_{j} \Omega_{j}^{*}+M_{j} \overline{\Omega_{j}^{*}}\right]}{\left(p^{2}-M_{i}^{2}\right)\left(p^{2}-M_{j}^{2}\right)^{2}}\right] \operatorname{Tr}\left[p_{l} . \sigma p . \bar{\sigma} p_{\Psi} . \sigma p . \bar{\sigma}\right] \\
& +4 \operatorname{Im}\left[y_{i} h_{i \alpha}^{*} y_{j}^{*} h_{j \alpha}\right]\left[\frac{\operatorname{Im}\left[p^{2}(\Xi)_{i}+M_{i}^{2}(\Xi)_{i}^{T}+M_{i} \Omega_{i}+M_{i} \overline{\Omega_{i}}\right]}{\left(p^{2}-M_{i}^{2}\right)^{2}\left(p^{2}-M_{j}^{2}\right)}\right] \operatorname{Tr}\left[p_{l} . \sigma p . \bar{\sigma} p_{\Psi} . \sigma p . \bar{\sigma}\right] \\
& +4 \operatorname{Im}\left[y_{i}^{*} h_{i \alpha}^{*} y_{j}^{*} h_{j \alpha}\right]\left[\frac{\operatorname{Im}\left[p^{2}(\Xi)_{j}^{*}+M_{j}^{2}\left(\Xi^{T}\right)_{j}^{*}+M_{j} \Omega_{j}+M_{j} \overline{\Omega_{j}}\right]}{\left(p^{2}-M_{i}^{2}\right)\left(p^{2}-M_{j}^{2}\right)^{2}}\right] M_{i} m_{\Psi} \operatorname{Tr}\left[p_{l} \cdot \sigma \bar{\sigma} \cdot p\right] \\
& +4 \operatorname{Im}\left[y_{i}^{*} h_{i \alpha}^{*} y_{j}^{*} h_{j \alpha}\right]\left[\frac{\operatorname{Im}\left[p^{2}\left(M_{i}(\Xi)_{i}^{T}+M_{i}(\Xi)_{i}+\Omega_{i}\right)+M_{i}^{2}(\bar{\Omega})_{i}\right]}{\left(p^{2}-M_{i}^{2}\right)^{2}\left(p^{2}-M_{j}^{2}\right)}\right] m_{\Psi} \operatorname{Tr}\left[p_{l} \cdot \sigma \bar{\sigma} \cdot p\right] \\
& +4 \operatorname{Im}\left[y_{i} h_{i \alpha}^{*} y_{j} h_{j \alpha}\right]\left[\frac{\operatorname{Im}\left[p^{2}\left[M_{j}(\Xi)_{j}^{*}+M_{j}\left(\Xi^{T}\right)_{j}^{*}+\Omega_{j}^{*}\right]+M_{j}^{2} \overline{\Omega_{j}^{*}}\right.}{\left.\left(p^{2}-M_{i}^{2}\right)\left(p^{2}-M_{j}^{2}\right)^{2}\right]}\right] \operatorname{Tr}\left[p_{l} \cdot \sigma \bar{\sigma} \cdot p\right] m_{\Psi} \\
& +4 \operatorname{Im}\left[y_{i} h_{i \alpha}^{*} y_{j} h_{j \alpha}\right]\left[\frac{\operatorname{Im}\left[p^{2}(\Xi)_{i}+M_{i}^{2}(\Xi)_{i}^{T}+M_{i} \Omega_{i}+M_{i} \overline{\Omega_{i}}\right]}{\left(p^{2}-M_{i}^{2}\right)^{2}\left(p^{2}-M_{j}^{2}\right)}\right] M_{j} m_{\Psi} \operatorname{Tr}\left[p_{l} \cdot \sigma \bar{\sigma} \cdot p\right]
\end{aligned}
$$

Note that for the full loop corrected propagators $\left(\Xi^{T}\right)^{*}=\Xi$ and $\bar{\Omega}=\Omega^{*}$. One can easily figure out that the asymmetry we have from the tree-loop interference calculation matches with the asymmetry we got in Eq, (A18) after replacing the resummed propagators.

\section{APPENDIX B: TWO BODY DECAY OF $N_{i}$}

The decay width for the decay $N_{1} \rightarrow \eta l$ is given by

$$
\Gamma_{N_{1} \rightarrow \eta l}=\frac{M_{1}}{8 \pi}\left(h^{\dagger} h\right)_{11}\left(1-\frac{m_{\eta}^{2}}{M_{1}^{2}}\right)^{2}
$$

The $C P$ asymmetry parameter for $N_{1} \rightarrow l_{i} \eta, \overline{l_{i}} \bar{\eta}$ is given by

$$
\begin{aligned}
\epsilon_{\left(N_{1}\right)_{i}}= & \frac{1}{8 \pi\left(h^{\dagger} h\right)_{11}}\left[f\left(\frac{M_{2}^{2}}{M_{1}^{2}}, \frac{m_{\eta}^{2}}{M_{1}^{2}}\right) \operatorname{Im}\left[h_{i 1}^{*} h_{i 2}\left(h^{\dagger} h\right)_{12}\right]\right. \\
& \left.-\frac{M_{1}^{2}}{M_{2}^{2}-M_{1}^{2}}\left(1-\frac{m_{\eta}^{2}}{M_{1}^{2}}\right)^{2} \operatorname{Im}\left[h_{i 1}^{*} h_{i 2} H_{12}\right]\right]
\end{aligned}
$$

where, the function $f\left(r_{j i}, \eta_{i}\right)$ is coming from the interference of the tree-level and one loop diagrams and has the form

$$
f\left(r_{j i}, \eta_{i}\right)=\sqrt{r_{j i}}\left[1+\frac{\left(1-2 \eta_{i}+r_{j i}\right)}{\left(1-\eta_{i}^{2}\right)^{2}} \ln \left(\frac{r_{j i}-\eta_{i}^{2}}{1-2 \eta_{i}+r_{j i}}\right)\right]
$$

with $r_{j i}=M_{j}^{2} / M_{i}^{2}$ and $\eta_{i}=m_{\eta}^{2} / M_{i}^{2}$. The self-energy contribution $H_{i j}$ is given by

$$
H_{i j}=\left(h^{\dagger} h\right)_{i j} \frac{M_{j}}{M_{i}}+\left(h^{\dagger} h\right)_{i j}^{*}
$$

Now, the $C P$ asymmetry parameter, neglecting the flavor effects (summing over final state flavors $\alpha$ ) is

$$
\epsilon_{N_{1}}=\frac{1}{8 \pi\left(h^{\dagger} h\right)_{11}} \operatorname{Im}\left[\left(\left(h^{\dagger} h\right)_{12}\right)^{2}\right] \frac{1}{\sqrt{r_{21}}} F\left(r_{21}, \eta_{1}\right)
$$

where the function $F\left(r_{j i}, \eta\right)$ is defined as

$$
F\left(r_{j i}, \eta_{i}\right)=\sqrt{r_{j i}}\left[f\left(r_{j i}, \eta_{i}\right)-\frac{\sqrt{r_{j i}}}{r_{j i}-1}\left(1-\eta_{i}\right)^{2}\right] .
$$


[1] M. Tanabashi et al. (Particle Data Group), Review of particle physics, Phys. Rev. D 98, 030001 (2018).

[2] N. Aghanim et al. (Planck Collaboration), Planck 2018 results. VI. Cosmological parameters, Astron. Astrophys. 641, A6 (2020).

[3] A. D. Sakharov, Violation of $C P$ Invariance, $C$ asymmetry, and baryon asymmetry of the universe, Usp. Fiz. Nauk 161, 61 (1991) [Pis'ma Zh. Eksp. Teor. Fiz. 5, 32 (1967)].

[4] S. Weinberg, Cosmological Production of Baryons, Phys. Rev. Lett. 42, 850 (1979).

[5] E. W. Kolb and S. Wolfram, Baryon number generation in the early universe, Nucl. Phys. B172, 224 (1980); B195, 542(E) (1982).

[6] V. A. Kuzmin, V. A. Rubakov, and M. E. Shaposhnikov, On the anomalous electroweak baryon number nonconservation in the early Universe, Phys. Lett. 155B, 36 (1985).

[7] M. Fukugita and T. Yanagida, Baryogenesis without grand unification, Phys. Lett. 174B, 45 (1986).

[8] S. Davidson, E. Nardi, and Y. Nir, Leptogenesis, Phys. Rep. 466, 105 (2008).

[9] P. Minkowski, $\mu \rightarrow e \gamma$ at a rate of one out of $10^{9}$ muon decays?, Phys. Lett. 67B, 421 (1977).

[10] R. N. Mohapatra and G. Senjanovic, Neutrino Mass and Spontaneous Parity Violation, Phys. Rev. Lett. 44, 912 (1980).

[11] T. Yanagida, Horizontal gauge symmetry and masses of neutrinos, Conf. Proc. C 7902131, 95 (1979).

[12] M. Gell-Mann, P. Ramond, and R. Slansky, Complex spinors and unified theories, Conf. Proc. C 790927, 315 (1979).

[13] S. L. Glashow, The future of elementary particle physics, NATO Sci. Ser. B 61, 687 (1980).

[14] J. Schechter and J. W. F. Valle, Neutrino masses in $\mathrm{SU}(2) \times \mathrm{U}(1)$ theories, Phys. Rev. D 22, 2227 (1980).

[15] F. Zwicky, Die Rotverschiebung von extragalaktischen Nebeln, Helv. Phys. Acta 6, 110 (1933); Gen. Relativ. Gravit. 41, 207 (2009).

[16] V. C. Rubin and W. K. Ford, Jr., Rotation of the andromeda nebula from a spectroscopic survey of emission regions, Astrophys. J. 159, 379 (1970).

[17] D. Clowe, M. Bradac, A. H. Gonzalez, M. Markevitch, S. W. Randall, C. Jones, and D. Zaritsky, A direct empirical proof of the existence of dark matter, Astrophys. J. 648, L109 (2006).

[18] E. W. Kolb and M. S. Turner, The early universe, Front. Phys. 69, 1 (1990).

[19] G. Arcadi, M. Dutra, P. Ghosh, M. Lindner, Y. Mambrini, M. Pierre, S. Profumo, and F. S. Queiroz, The waning of the WIMP? A review of models, searches, and constraints, Eur. Phys. J. C 78, 203 (2018).

[20] E. Ma, Verifiable radiative seesaw mechanism of neutrino mass and dark matter, Phys. Rev. D 73, 077301 (2006).

[21] T. Hambye, F. S. Ling, L. Lopez Honorez, and J. Rocher, Scalar multiplet dark matter, J. High Energy Phys. 07 (2009) 090; 05 (2010) 066(E).

[22] J. Racker, Mass bounds for baryogenesis from particle decays and the inert doublet model, J. Cosmol. Astropart. Phys. 03 (2014) 025.
[23] J. D. Clarke, R. Foot, and R. R. Volkas, Natural leptogenesis and neutrino masses with two Higgs doublets, Phys. Rev. D 92, 033006 (2015).

[24] T. Hugle, M. Platscher, and K. Schmitz, Low-scale leptogenesis in the scotogenic neutrino mass model, Phys. Rev. D 98, 023020 (2018).

[25] D. Borah, P. S. B. Dev, and A. Kumar, TeV scale leptogenesis, inflaton dark matter and neutrino mass in a scotogenic model, Phys. Rev. D 99, 055012 (2019).

[26] D. Mahanta and D. Borah, Fermion dark matter with $\mathrm{N}_{2}$ leptogenesis in minimal scotogenic model, J. Cosmol. Astropart. Phys. 11 (2019) 021.

[27] D. Mahanta and D. Borah, TeV scale leptogenesis with dark matter in non-standard cosmology, J. Cosmol. Astropart. Phys. 04 (2020) 032.

[28] L. Sarma, P. Das, and M. K. Das, Scalar dark matter, leptogenesis and $0 \nu \beta \beta$ in minimal scotogenic model, Nucl. Phys. B963, 115300 (2021).

[29] S. Kashiwase and D. Suematsu, Baryon number asymmetry and dark matter in the neutrino mass model with an inert doublet, Phys. Rev. D 86, 053001 (2012).

[30] S. Kashiwase and D. Suematsu, Leptogenesis and dark matter detection in a TeV scale neutrino mass model with inverted mass hierarchy, Eur. Phys. J. C 73, 2484 (2013).

[31] S. Davidson and A. Ibarra, A lower bound on the righthanded neutrino mass from leptogenesis, Phys. Lett. B 535, 25 (2002).

[32] A. Masiero and A. Riotto, Cosmic delta B from lepton violating interactions at the electroweak phase transition, Phys. Lett. B 289, 73 (1992).

[33] R. Adhikari and U. Sarkar, Baryogenesis in a supersymmetric model without R-parity, Phys. Lett. B 427, 59 (1998).

[34] U. Sarkar and R. Adhikari, Baryogenesis through R-parity violation, Phys. Rev. D 55, 3836 (1997).

[35] T. Hambye, Leptogenesis at the TeV scale, Nucl. Phys. B633, 171 (2002).

[36] A. Dasgupta, P. B. Dev, S. K. Kang, and Y. Zhang, New mechanism for matter-antimatter asymmetry and connection with dark matter, Phys. Rev. D 102, 055009 (2020).

[37] W. Abdallah, A. Kumar, and A. K. Saha, Soft leptogenesis in the NMSSM with a singlet right-handed neutrino superfield, J. High Energy Phys. 04 (2020) 065.

[38] Y. Grossman, T. Kashti, Y. Nir, and E. Roulet, Leptogenesis from Supersymmetry Breaking, Phys. Rev. Lett. 91, 251801 (2003).

[39] G. D’Ambrosio, G. F. Giudice, and M. Raidal, Soft leptogenesis, Phys. Lett. B 575, 75 (2003).

[40] C. S. Fong, M. Gonzalez-Garcia, and E. Nardi, Leptogenesis from soft supersymmetry breaking (soft leptogenesis), Int. J. Mod. Phys. A 26, 3491 (2011).

[41] D. Borah and R. Adhikari, Abelian gauge extension of standard model: Dark matter and radiative neutrino mass, Phys. Rev. D 85, 095002 (2012).

[42] R. Adhikari, D. Borah, and E. Ma, New U(1) gauge model of radiative lepton masses with sterile neutrino and dark matter, Phys. Lett. B 755, 414 (2016).

[43] D. Nanda and D. Borah, Common origin of neutrino mass and dark matter from anomaly cancellation requirements of a $U(1)_{B-L}$ model, Phys. Rev. D 96, 115014 (2017). 
[44] B. Barman, D. Borah, P. Ghosh, and A. K. Saha, Flavoured gauge extension of singlet-doublet fermionic dark matter: Neutrino mass, high scale validity and collider signatures, J. High Energy Phys. 10 (2019) 275.

[45] A. Biswas, D. Borah, and D. Nanda, Type III seesaw for neutrino masses in $\mathrm{U}(1)_{B-L}$ model with multi-component dark matter, J. High Energy Phys. 12 (2019) 109.

[46] D. Nanda and D. Borah, Connecting light dirac neutrinos to a multi-component dark matter scenario in gauged $B-L$ model, Eur. Phys. J. C 80, 557 (2020).

[47] A. Merle and M. Platscher, Running of radiative neutrino masses: the scotogenic model, revisited, J. High Energy Phys. 11 (2015) 148.

[48] P. F. de Salas, D. V. Forero, C. A. Ternes, M. Tortola, and J. W. F. Valle, Status of neutrino oscillations 2018: $3 \sigma$ hint for normal mass ordering and improved $C P$ sensitivity, Phys. Lett. B 782, 633 (2018).

[49] I. Esteban, M. C. Gonzalez-Garcia, A. Hernandez-Cabezudo, M. Maltoni, and T. Schwetz, Global analysis of three-flavour neutrino oscillations: Synergies and tensions in the determination of $\theta_{2} 3, \delta_{C} P$, and the mass ordering, J. High Energy Phys. 01 (2019) 106.

[50] J. A. Casas and A. Ibarra, Oscillating neutrinos and $\mu \rightarrow e$, $\gamma$, Nucl. Phys. B618, 171 (2001).

[51] T. Toma and A. Vicente, Lepton flavor violation in the scotogenic model, J. High Energy Phys. 01 (2014) 160.

[52] A. Ibarra and G. G. Ross, Neutrino phenomenology: The case of two right-handed neutrinos, Phys. Lett. B 591, 285 (2004).

[53] E. Lundstrom, M. Gustafsson, and J. Edsjo, The inert doublet model and LEP II limits, Phys. Rev. D 79, 035013 (2009).

[54] M. Aaboud et al. (ATLAS Collaboration), Combination of Searches for Invisible Higgs Boson Decays with the ATLAS Experiment, Phys. Rev. Lett. 122, 231801 (2019).

[55] ATLAS Collaboration, Search for invisible Higgs boson decays with vector boson fusion signatures with the ATLAS detector using an integrated luminosity of $139 \mathrm{fb}^{-1}, \quad$ (2020), https://cds.cern.ch/record/2715447? $\ln =$ en.

[56] D. Borah and A. Dasgupta, Left right symmetric models with a mixture of keV-TeV dark matter, J. Phys. G 46, 105004 (2019).

[57] X. Miao, S. Su, and B. Thomas, Trilepton signals in the inert doublet model, Phys. Rev. D 82, 035009 (2010).

[58] M. Gustafsson, S. Rydbeck, L. Lopez-Honorez, and E. Lundstrom, Status of the inert doublet model and the role of multileptons at the LHC, Phys. Rev. D 86, 075019 (2012).

[59] A. Datta, N. Ganguly, N. Khan, and S. Rakshit, Exploring collider signatures of the inert Higgs doublet model, Phys. Rev. D 95, 015017 (2017).

[60] P. Poulose, S. Sahoo, and K. Sridhar, Exploring the inert doublet model through the dijet plus missing transverse energy channel at the LHC, Phys. Lett. B 765, 300 (2017).

[61] M. Hashemi and S. Najjari, Observability of inert scalars at the LHC, Eur. Phys. J. C 77, 592 (2017).

[62] A. Belyaev, G. Cacciapaglia, I. P. Ivanov, F. Rojas, and M. Thomas, Anatomy of the inert two Higgs doublet model in the light of the LHC and non-LHC dark matter searches, Phys. Rev. D 97, 035011 (2018).

[63] A. Belyaev, T. F. P. Tomei, P. Mercadante, C. Moon, S. Moretti, S. Novaes, L. Panizzi, F. Rojas, and M. Thomas, Advancing LHC probes of dark matter from the inert twoHiggs-doublet model with the monojet signal, Phys. Rev. D 99, 015011 (2019).

[64] D. Borah, S. Sadhukhan, and S. Sahoo, Lepton portal limit of inert Higgs doublet dark matter with radiative neutrino mass, Phys. Lett. B 771, 624 (2017).

[65] D. Borah, D. Nanda, N. Narendra, and N. Sahu, Right-handed neutrino dark matter with radiative neutrino mass in gauged $B-L$ model, Nucl. Phys. B950, 114841 (2020).

[66] M. Sher, Electroweak Higgs potentials and vacuum stability, Phys. Rep. 179, 273 (1989).

[67] K. Kannike, Vacuum stability conditions from copositivity criteria, Eur. Phys. J. C 72, 2093 (2012).

[68] J. Chakrabortty, P. Konar, and T. Mondal, Copositive criteria and boundedness of the scalar potential, Phys. Rev. D 89, 095008 (2014).

[69] D. Borah, A. Dasgupta, and S. K. Kang, Leptogenesis from dark matter annihilations in scotogenic model, Eur. Phys. J. C 80, 498 (2020).

[70] D. Borah, A. Dasgupta, and S. K. Kang, Two-component dark matter with cogenesis of the baryon asymmetry of the Universe, Phys. Rev. D 100, 103502 (2019).

[71] W.-C. Huang, H. Päs, and S. Zeiüner, Scalar dark matter, GUT baryogenesis and radiative neutrino mass, Phys. Rev. D 98, 075024 (2018).

[72] S. Bhattacharya, P. Ghosh, A. K. Saha, and A. Sil, Two component dark matter with inert Higgs doublet: Neutrino mass, high scale validity and collider searches, J. High Energy Phys. 03 (2020) 090.

[73] Q.-H. Cao, E. Ma, J. Wudka, and C. P. Yuan, Multipartite dark matter, arXiv:0711.3881.

[74] K. M. Zurek, Multi-component dark matter, Phys. Rev. D 79, 115002 (2009).

[75] D. Chialva, P. S. B. Dev, and A. Mazumdar, Multiple dark matter scenarios from ubiquitous stringy throats, Phys. Rev. D 87, 063522 (2013).

[76] J. Heeck and H. Zhang, Exotic charges, multicomponent dark matter and light sterile neutrinos, J. High Energy Phys. 05 (2013) 164.

[77] A. Biswas, D. Majumdar, A. Sil, and P. Bhattacharjee, Two component dark matter: A possible explanation of $130 \mathrm{GeV} \gamma$ - ray Line from the galactic centre, J. Cosmol. Astropart. Phys. 12 (2013) 049.

[78] S. Bhattacharya, A. Drozd, B. Grzadkowski, and J. Wudka, Two-component dark matter, J. High Energy Phys. 10 (2013) 158.

[79] L. Bian, R. Ding, and B. Zhu, Two component Higgsportal dark matter, Phys. Lett. B 728, 105 (2014).

[80] L. Bian, T. Li, J. Shu, and X.-C. Wang, Two component dark matter with multi-Higgs portals, J. High Energy Phys. 03 (2015) 126.

[81] S. Esch, M. Klasen, and C. E. Yaguna, A minimal model for two-component dark matter, J. High Energy Phys. 09 (2014) 108. 
[82] A. Karam and K. Tamvakis, Dark matter and neutrino masses from a scale-invariant multi-Higgs portal, Phys. Rev. D 92, 075010 (2015).

[83] A. Karam and K. Tamvakis, Dark matter from a classically scale-invariant $S U(3)_{X}$, Phys. Rev. D 94, 055004 (2016).

[84] A. DiFranzo and G. Mohlabeng, Multi-component dark matter through a radiative Higgs portal, J. High Energy Phys. 01 (2017) 080.

[85] S. Bhattacharya, P. Poulose, and P. Ghosh, Multipartite Interacting Scalar Dark Matter in the light of updated LUX data, J. Cosmol. Astropart. Phys. 04 (2017) 043.

[86] A. D. Banik, M. Pandey, D. Majumdar, and A. Biswas, Two component WIMP-FImP dark matter model with singlet fermion, scalar and pseudo scalar, Eur. Phys. J. C 77, 657 (2017).

[87] M. Klasen, F. Lyonnet, and F. S. Queiroz, NLO+NLL collider bounds, Dirac fermion and scalar dark matter in the B-L model, Eur. Phys. J. C 77, 348 (2017).

[88] P. Ghosh, A. K. Saha, and A. Sil, Study of electroweak vacuum stability from extended Higgs portal of dark matter and neutrinos, Phys. Rev. D 97, 075034 (2018).

[89] A. Ahmed, M. Duch, B. Grzadkowski, and M. Iglicki, Multi-component dark matter: The vector and fermion case, Eur. Phys. J. C 78, 905 (2018).

[90] S. Bhattacharya, P. Ghosh, T. N. Maity, and T. S. Ray, Mitigating direct detection bounds in non-minimal Higgs portal scalar dark matter models, J. High Energy Phys. 10 (2017) 088.

[91] S. Bhattacharya, A. K. Saha, A. Sil, and J. Wudka, Dark matter as a remnant of SQCD inflation, J. High Energy Phys. 10 (2018) 124

[92] S. Bhattacharya, P. Ghosh, and N. Sahu, Multipartite dark matter with scalars, fermions and signatures at LHC, J. High Energy Phys. 02 (2019) 059.

[93] M. Aoki and T. Toma, Boosted self-interacting dark matter in a multi-component dark matter model, J. Cosmol. Astropart. Phys. 10 (2018) 020.

[94] A. D. Banik, A. K. Saha, and A. Sil, Scalar assisted singlet doublet fermion dark matter model and electroweak vacuum stability, Phys. Rev. D 98, 075013 (2018).

[95] B. Barman, S. Bhattacharya, and M. Zakeri, Multipartite dark matter in $S U(2)_{N}$ extension of standard model and signatures at the LHC, J. Cosmol. Astropart. Phys. 09 (2018) 023.

[96] S. Y. Ayazi and A. Mohamadnejad, Scale-invariant two component dark matter, Eur. Phys. J. C 79, 140 (2019).

[97] A. Poulin and S. Godfrey, Multi-component dark matter from a hidden gauged SU(3), Phys. Rev. D 99, 076008 (2019).

[98] S. Chakraborti and P. Poulose, Interplay of scalar and fermionic components in a multi-component dark matter scenario, Eur. Phys. J. C 79, 420 (2019).

[99] S. Chakraborti, A. D. Banik, and R. Islam, Probing multicomponent extension of inert doublet model with a vector dark matter, Eur. Phys. J. C 79, 662 (2019).

[100] N. Bernal, D. Restrepo, C. Yaguna, and Ó. Zapata, Twocomponent dark matter and a massless neutrino in a new B - L model, Phys. Rev. D 99, 015038 (2019).

[101] F. Elahi and S. Khatibi, Multi-component dark matter in a non-abelian dark sector, Phys. Rev. D 100, 015019 (2019).
[102] D. Borah, R. Roshan, and A. Sil, Minimal two-component scalar doublet dark matter with radiative neutrino mass, Phys. Rev. D 100, 055027 (2019).

[103] S. Bhattacharya, N. Chakrabarty, R. Roshan, and A. Sil, Multicomponent dark matter in extended $U(1)_{B-L}$ : Neutrino mass and high scale validity, J. Cosmol. Astropart. Phys. 04 (2020) 013.

[104] D. Borah, S. Mahapatra, D. Nanda, and N. Sahu, Inelastic fermion dark matter origin of XENON1T excess with muon $(g-2)$ and light neutrino mass, Phys. Lett. B 811, 135933 (2020).

[105] P. Gondolo and G. Gelmini, Cosmic abundances of stable particles: Improved analysis, Nucl. Phys. B360, 145 (1991).

[106] N. G. Deshpande and E. Ma, Pattern of symmetry breaking with two Higgs doublets, Phys. Rev. D 18, 2574 (1978).

[107] A. Dasgupta and D. Borah, Scalar dark matter with type II seesaw, Nucl. Phys. B889, 637 (2014).

[108] M. Cirelli, N. Fornengo, and A. Strumia, Minimal dark matter, Nucl. Phys. B753, 178 (2006).

[109] R. Barbieri, L. J. Hall, and V. S. Rychkov, Improved naturalness with a heavy Higgs: An alternative road to LHC physics, Phys. Rev. D 74, 015007 (2006).

[110] E. Ma, Common origin of neutrino mass, dark matter, and baryogenesis, Mod. Phys. Lett. A 21, 1777 (2006).

[111] L. Lopez Honorez, E. Nezri, J. F. Oliver, and M. H. G. Tytgat, The inert doublet model: An archetype for dark matter, J. Cosmol. Astropart. Phys. 02 (2007) 028.

[112] E. M. Dolle and S. Su, The inert dark matter, Phys. Rev. D 80, 055012 (2009).

[113] L. Lopez Honorez and C. E. Yaguna, The inert doublet model of dark matter revisited, J. High Energy Phys. 09 (2010) 046.

[114] L. Lopez Honorez and C. E. Yaguna, A new viable region of the inert doublet model, J. Cosmol. Astropart. Phys. 01 (2011) 002.

[115] A. Goudelis, B. Herrmann, and O. Stal, Dark matter in the inert doublet model after the discovery of a Higgslike boson at the LHC, J. High Energy Phys. 09 (2013) 106.

[116] A. Arhrib, Y.-L. S. Tsai, Q. Yuan, and T.-C. Yuan, An updated analysis of inert Higgs doublet model in light of the recent results from LUX, PLANCK, AMS-02 and LHC, J. Cosmol. Astropart. Phys. 06 (2014) 030.

[117] M. A. Daz, B. Koch, and S. Urrutia-Quiroga, Constraints to dark matter from inert Higgs doublet model, Adv. High Energy Phys. 2016, 8278375 (2016).

[118] A. Ahriche, A. Jueid, and S. Nasri, Radiative neutrino mass and Majorana dark matter within an inert Higgs doublet model, Phys. Rev. D 97, 095012 (2018).

[119] D. Borah and A. Gupta, New viable region of an inert Higgs doublet dark matter model with scotogenic extension, Phys. Rev. D 96, 115012 (2017).

[120] K. Griest and D. Seckel, Three exceptions in the calculation of relic abundances, Phys. Rev. D 43, 3191 (1991).

[121] V. Silveira and A. Zee, Scalar phantoms, Phys. Lett. 161B, 136 (1985).

[122] J. McDonald, Gauge singlet scalars as cold dark matter, Phys. Rev. D 50, 3637 (1994). 
[123] C. P. Burgess, M. Pospelov, and T. ter Veldhuis, The Minimal model of nonbaryonic dark matter: A singlet scalar, Nucl. Phys. B619, 709 (2001).

[124] P. Athron et al. (GAMBIT Collaboration), Status of the scalar singlet dark matter model, Eur. Phys. J. C 77, 568 (2017).

[125] D. Borah, R. Roshan, and A. Sil, Sub-TeV singlet scalar dark matter and electroweak vacuum stability with vectorlike fermions, Phys. Rev. D 102, 075034 (2020).

[126] G. B. langer, F. Boudjema, A. Pukhov, and A. Semenov, micrOMEGAs4.1: Two dark matter candidates, Comput. Phys. Commun. 192, 322 (2015).

[127] A. Alloul, N. D. Christensen, C. Degrande, C. Duhr, and B. Fuks, FeynRules 2.0-A complete toolbox for tree-level phenomenology, Comput. Phys. Commun. 185, 2250 (2014).

[128] A. Belyaev, N. D. Christensen, and A. Pukhov, CalcHEP 3.4 for collider physics within and beyond the standard model, Comput. Phys. Commun. 184, 1729 (2013).

[129] E. Aprile et al. (XENON Collaboration), First Dark Matter Search Results from the XENON1T Experiment, Phys. Rev. Lett. 119, 181301 (2017).

[130] E. Aprile et al., Dark Matter Search Results from a One Tonne $\times$ Year Exposure of XENON1T, Phys. Rev. Lett. 121, 111302 (2018).

[131] D. S. Akerib et al. (LUX Collaboration), Results from a Search for Dark Matter in the Complete LUX Exposure, Phys. Rev. Lett. 118, 021303 (2017).

[132] A. Tan et al. (PandaX-II Collaboration), Dark Matter Results from First 98.7 Days of Data from the PandaXII Experiment, Phys. Rev. Lett. 117, 121303 (2016).

[133] X. Cui et al. (PandaX-II Collaboration), Dark Matter Results From 54-Ton-Day Exposure of PandaX-II Experiment, Phys. Rev. Lett. 119, 181302 (2017).

[134] J. Herrero-Garcia, A. Scaffidi, M. White, and A. G. Williams, On the direct detection of multi-component dark matter: Sensitivity studies and parameter estimation, J. Cosmol. Astropart. Phys. 11 (2017) 021.

[135] J. Herrero-Garcia, A. Scaffidi, M. White, and A. G. Williams, Time-dependent rate of multicomponent dark matter: Reproducing the DAMA/LIBRA phase-2 results, Phys. Rev. D 98, 123007 (2018).

[136] A. Abada, S. Davidson, F.-X. Josse-Michaux, M. Losada, and A. Riotto, Flavor issues in leptogenesis, J. Cosmol. Astropart. Phys. 04 (2006) 004.

[137] A. Abada, S. Davidson, A. Ibarra, F. X. Josse-Michaux, M. Losada, and A. Riotto, Flavour matters in leptogenesis, J. High Energy Phys. 09 (2006) 010.

[138] E. Nardi, Y. Nir, E. Roulet, and J. Racker, The importance of flavor in leptogenesis, J. High Energy Phys. 01 (2006) 164.

[139] S. Blanchet and P. Di Bari, Flavor effects on leptogenesis predictions, J. Cosmol. Astropart. Phys. 03 (2007) 018.

[140] P. S. B. Dev, P. Di Bari, B. Garbrecht, S. Lavignac, P. Millington, and D. Teresi, Flavor effects in leptogenesis, Int. J. Mod. Phys. A 33, 1842001 (2018).

[141] T. Alanne, T. Hugle, M. Platscher, and K. Schmitz, Lowscale leptogenesis assisted by a real scalar singlet, J. Cosmol. Astropart. Phys. 03 (2019) 037.

[142] A. Gando et al. (KamLAND-Zen Collaboration), Search for Majorana Neutrinos near the Inverted Mass Hierarchy Region with KamLAND-Zen, Phys. Rev. Lett. 117, 082503 (2016); 117, 109903(A) (2016).

[143] A. Vicente and C. E. Yaguna, Probing the scotogenic model with lepton flavor violating processes, J. High Energy Phys. 02 (2015) 144.

[144] D. Borah, A. Dasgupta, K. Fujikura, S. K. Kang, and D. Mahanta, Observable gravitational waves in minimal scotogenic model, J. Cosmol. Astropart. Phys. 08 (2020) 046.

[145] H. K. Dreiner, H. E. Haber, and S. P. Martin, Two-component spinor techniques and Feynman rules for quantum field theory and supersymmetry, Phys. Rep. 494, 1 (2010). 